\title{
The Hong Kong Symposium on Paediatric Cardiology and Paediatric Cardiac Surgery
}

\author{
Organized by Maurice P. Leung and C. K. Mok
}

Hong Kong, November 1992

\author{
Replacement of the mitral valve in children \\ Richard A. Jonas \\ Children's Hospital, Boston
}

In the past, the most common indication for replacement of the left atrioventricular valve in children was regurgitation following repair of complete atrioventricular septal defect. An improved understanding of methods of valvoplasty as part of the repair of this lesion has dramarically reduced the need for replacement of the left valve in this setting. Furthermore, because of the left ventricular and left atrial dilatation which is a usual accompaniment of a regurgitant mitral valve, replacement of a mitral valve even in the infant with mitral regurgitation is generally not a serious technical problem.

In contrast to the patient with mitral regurgitation, the young infant with congenital mitral stenosis presents a serious problem when valvar replacement is required. Careful assessment must be made of the adequacy of the left ventricle, the left ventricular outflow tract including the attachments of the leaflets of the aortic valve, as well as the ascending aorta and aortic arch, since these are often hypoplastic as part of Shone's syndrome. Balloon dilatation of the mitral valve has been successfully applied and, even if mitral regurgitation results, this may be beneficial since there is subsequent dilatation of the left ventricle and atrium. If valvar replacement is ultimately required, it is rarely possible to place even the smallest prosthesis in an annular position. The annulus itself is often no more than 10 to $12 \mathrm{~mm}$ in diameter. We have found that placement of the prosthesis completely within the left atrium is a reasonable compromise. Approach is through a vertical transatrial septal incision following a right atriotomy. The prosthesis is positioned immediately apical relative to the orifices of the inferior pulmonary veins. Anteriorly, sutures are passed through the atrial septum. Supraannular placement of the mitral valve avoids insertion of sutures in the region of the conduction tissues. It also avoids tension on the mitral annulus itself which may cause complete heart block even in the absence of direct placement of sutures into the conduction system. Recent experience has suggested that the St Jude prosthesis is the most suitable for this specific application because the leaflets remain protected by the valvar housing. This is in contrast to the modified $16 \mathrm{~mm}$ and $18 \mathrm{~mm}$ Carbomedics valves, in which projection of the leaflets beyond the ventricular edge of the housing may result in compromised motion. Anticoagulation with Coumadin in infants and young children has not proven to be problematic.

Berween 1973 and 1987, 25 patients underwent replacement of the mitral valve in the first year of life. Five patients had mitral stenosis and 20 had mitral regurgitation. Early mortality was high in the first years of the study, but was zero between 1983 and 1987. Actuarial freedom from repeat valvar replacement was $45 \%$ at 3 years and remained unchanged to 5 years. Up to 1987,4 patients had had supraannular replacements with no deaths.

Surgical technique of rapid two stage arterial switch procedure Richard A. Jonas Children's Hospital, Boston

Patients who present beyond the neonatal period with complete transposition and intact ventricular septum with left ventricular pressures less than 60 to $70 \%$ of pressures in the right ventricle require preparation of the left ventricle before proceeding to an arterial switch procedure. Both laboratory data and echo studies of athletes in training have demonstrated the extremely rapid growth of myocardium in response to a pressure load. Although the operative approach was originally through a right thoracotomy, we currently undertake the first stage preparatory procedure through a median sternotomy. The origin of the right subclavian artery is dissected free as is the right pulmonary artery. A modified $3.5 \mathrm{~mm}$ right Blalock shunt is constructed. A Dacron impregnated silastic band is placed around the pulmonary trunk and is tightened so as to achieve a left ventricular pressure between 70 and $90 \%$ systemic.

Approximately one third of patients will require considerable inotropic support in the interval of approximately one week before the arterial switch procedure is undertaken. These patients also require ventilation throughout this interval. There may be a considerable requirement for bicarbonate in the first hours after the procedure, as the right ventricle adapts to the sudden increase in volume load and the left ventricle responds to an increased pressure load. Approximately one third of patients will require moderate inotropic support and the final one third of patients may be extubated one to two days after the surgery and have an uneventful interval period.

Echocardiography has documented rapid growth of myocardium in the interval period. Ventricular function generally deteriorates for 2 to 3 days, but returns to baseline levels by 6 to 7 days after the preparatory procedure.

The technique of the arterial switch procedure is unchanged from that used as a primary procedure other than division and oversewing of the shunt and removal of the band around the pulmonary trunk. The brevicy of the interval period will hopefully decrease permanent distortion of the neoaortic valve, though a relatively high incidence 
of trivial to mild aortic regurgitation has been observed by color Doppler echocardiography.

Up to July, 1990, 25 patients underwent the first stage of a rapid two stage arterial switch procedure at Children's Hospital, Boston. Median age at the time of the arterial switch was 4 months. There was one death during the interval between palliation and correction in a child who had previously undergone repair of aortic coarctation. One child had a Senning procedure when he was found to have a long intramural course of the left coronary artery. The arterial switch procedure was carried out in the remaining 23 children, with one death in a child who had a preoperative history of aortic thrombosis. Thus, combined early mortality for the two procedures and the interval period was $8 \%$. There was one late death 6 months postoperatively in a child with a single coronary artery. The mean follow-up of the 21 long-term survivors was 17 months. There was one reoperation for bilateral stenoses of the pulmonary arteries.

Staged repair of patients with one functioning ventricle Richard A. Jonas

Children's Hospital, Boston

Palliation of the child with a functionally single ventricle with either a shunt or a banded pulmonary trunk is inherently inefficient because of the mixing of pulmonary and systemic venous return and, therefore, recirculation of at least some of both the pulmonary and systemic venous returns to the vascular bed. Following construction of either a bidirectional Glenn shunt or a Fontan procedure, this inefficiency is largely eliminated because pulmonary blood flow is derived entirely from systemic venous return. There are important differences, however, between the bidirectional Glenn shunt and the Fontan procedure, mainly related to the lower systemic venous pressure to which abdominal organs will be exposed following the bidirectional Glenn shunt relative to the Fontan procedure. Therefore, if an extensive reconstructive procedure is required, such as pulmonary arterial reconstruction or relief of intracardiac systemic outflow obstruction, the bidirectional Glenn shunt can be used as an intermediate procedure prior to proceeding to a Fontan procedure.

The Fontan procedure itself may be staged by fenestration of the intra-atrial baffle. The fenestration allows right-to-left flow, thereby decompressing the right atrium and preventing excessive elevation of systemic venous pressure, as well as allowing the maintenance of cardiac output at the expense of some degree of cyanosis. When ventricular function has improved, or pulmonary vascular resistance has decreased, there will be less shunting across the fenestration. The fenestration may close spontaneously. Alternatively, if satisfactory hemodynamics are demonstrated by temporary balloon occlusion 6 to 12 months postoperatively, closure may be undertaken in the catheterization laboratory using a double clamshell device.

Following the introduction of the bidirectional Glenn shunt as an intermediate step in 1988, and introduction of the fenestrated Fontan procedure in 1989, the mortality for Fontan procedures at Children's Hospital decreased to $6 \%$ in 1989 and $3 \%$ in 1990 . The population of patients in these years included many at high risk, including those with heterotaxy and hypoplastic left heart syndrome.

Total correction of tetralogy with pulmonary atresia Richard A. Jonas

Children's Hospital, Boston

The current philosophy of management of tetralogy of Fallot with pulmonary atresia is that, the earlier one establishes blood flow through the true central pulmonary arteries, the more likely there will be normal alveolar and pulmonary arterial development. This can be achieved only be close collaboration between the surgical and interventional cardiology teams. Management decisions are complicated by the variable development of aortopulmonary collateral vessels. The starting point for management involves detailed anatomical definition early in infancy of the distribution of the true pulmonary arteries and the aortopulmonary collateral vessels.

In the group of patients with ductal dependent true pulmonary arteries without important aortopulmonary collateral vessels, the patient is able to derive adequate flow of pulmonary blood through the true pulmonary arteries alone. In this setting, correction in the neonatal period may be undertaken with an extremely low probability that right ventricular pressure will be excessively elevated following closure of the ventricular septal defect and placement of a homograft from the right ventricle to the pulmonary trunk.

In the group of patients with severely hypoplastic central pulmonary arteries associated with multiple aortopulmonary collateral vessels, it is our policy to establish continuity early in infancy between the right ventricle and true central pulmonary arteries using a small homograft. Prior to this procedure, aortopulmonary collateral vessels which duplicate the distribution of the true central pulmonary arteries may be embolized using coils if this can be done without compromising arterial oxygen saturation. Within two to three months of placement of the homograft, repeat catheterization is undertaken, peripheral pulmonary arterial stenoses are dilated, and additional collateral vessels may be embolized. Several catheterization procedures may be required for the same purposes in the coming months. When a left-to-right shunt has developed, the ventricular septal defect may be closed.

In the group of patients with discontinuous pulmonary arteries and large collateral vessels which do not duplicate true central pulmonary arteries, the discontinuous pulmonary arteries should be brought into continuity as early in life as is feasible, although preliminary construction of a shunt may be necessary. When the true pulmonary arteries have been developed by placement of a homograft from the right ventricle, unifocalization procedures can be undertaken, generally through a thoracotomy.

In those patients in which the dimensions of the central pulmonary arteries are considered borderline, and doubt exists whether the true pulmonary arteries are adequate to take the complete cardiac output, one option is to close the ventricular septal defect with a Dacron patch which is fenestrated centrally.

When adequate growth of the pulmonary arteries has occurred, possibly aided by balloon dilatation, the fenestration can be closed with a clamshell device thereby avoiding further surgery.

Between 1984 and 1990, 27 patients (11 less than 9 months of age, 6 neonates) with tetralogy of Fallot with pulmonary atresia with diminutive continuous pulmonary arteries underwent placement of a homograft between the right ventricle and the junction of the diminutive right and left pulmonary arteries. Six patients died early or late. In 9 patients, unifocalization procedures were carried out at various times. Coil embolization and dilatation of peripheral pulmonary arterial stenoses were undertaken in the majority of patients. Of the 21 survivors, 10 have undergone closure of the ventricular septal defect with no deaths at a mean follow-up of 31 months. Of 11 patients not yet repaired, 2 are considered irreparable because of inadequate distal pulmonary arteries, 3 await unifocalization, and 6 are candidates for repair. Over the same time period, 7 patients have undergone management of diminutive and discontinuous central pulmonary arteries. The ventricular septal defect has been closed in 2 of the survivors following a number of intermediate procedures.

Diagnostic evaluation of pulmonary atresia and intact

ventricular septum

Maurice Leung

Grantham Hospital, Hong Kong

The diagnosis of pulmonary atresia and intact ventricular septum 
should be considered in any cyanotic neonate. Apart from the typical findings on physical examination, chest radiograph and electro-echocardiogram, a definitive diagnosis can be achieved using high resolution echocardiography. In a prospective study carried out between January 1982 and June 1987 at the Grantham Hospital, Hong Kong, 27 consecutive neonates suspected to be suffering from pulmonary atresia and intact ventricular septum underwent detailed cross-sectional echocardiographic examination before cardiac catheterization. Twenty-five of the 27 neonates had pulmonary atresia. In all 25 neonates, the diagnosis and right ventricular morphology assessed on the tripartite approach were correctly established by echocardiography. Associated Ebstein's malformation of the tricuspid valve was also correctly identified non-invasively in 2 babies. Among the 5 babies who had fistulous communications with the coronary arteries, echocardiography demonstrated a fistula in 1 and provided clues for its diagnosis in 2 others. For all the 25 neonates, echocardiographic dimensions of the tricuspid annulus, right ventricular infundibulum and pulmonary truncal diameter correlated well with the angiographic measurements. In a subsequent study carried out between January 1988 and June 1990, 12 babies with pulmonary atresia and intact ventricular septum were diagnosed. All babies immediately underwent closed pulmonary valvotomy when echocardiography revealed a right ventricle with representation of all three components, adequate inflow and outflow dimensions, and without fistulous communications to the coronary arterias. Post-mortem examination in 3 non-survivors, and angiocardiography performed on the 9 survivors at 5 to 18 months after valvotomy, confirmed the echocardiographic assessment of the right ventricular morphology. The combined results of this study suggested that, in the management of neonates with pulmonary atresia and intact ventricular septum, pre-operative evaluation by echocardiography is usually sufficient. Cardiac cathererization should be reserved for cases with obliteration of one or two components of the right ventricle, and to perform balloon valvoplasty at a later stage for any residual pulmonary stenosis following the neonatal valvotomy.

\section{Protocols for treatment of tetralogy of Fallot}

\section{Maurice Leung}

Grantham Hospital, Hong Kong

The mainstay of treatment for Tetralogy of Fallot is surgery. Medical treatment is usually a temporary measure instituted either to maintain ductal patency for severe neonatal cyanosis, or to relieve hypercyanotic spells. The operative protocol for any symptomatic baby is chiefly to decide on primary or staged repair. Primary repair must be considered psychologically, sociologically and economically advantageous. Thus, for centres well-experienced in cardiac surgery for young infants, total surgical correction would be performed for a baby presenting at or after 2 years of age. Such a patient should have large left and right pulmonary arterial diameters with a predicted late post-repair right to left ventricular pressure ratio below about 0.85 (according to prediction by Blackstone et al, with or without use of a transjunctional patch). With younger patients (less than 2 years of age), staged repair has been found to be safer when compared with those who required a transjunctional patch for their primary repair. Thus, the recommended palliation for these babies would be a modified Blalock-Taussig shunt. Surgical correction is then deferred till early childhood. Because young age was also found to be an incremental risk factor for operation on tetralogy of Fallot, there are centres which would prefer to perform total correction for these children at 3 to 4 years of age. With increasing experience and better technique for myocardial protection in infants, however, primary correction (with or without a transjunctional patch) has now been shown to carry a low mortality for young infants less than 1 year old. This must be the ultimate goal for operative treatment. The ones contraindicated for early primary repair would be those with anomalous origin of a coronary artery crossing the right ventricular outflow tract and those with the so-called absent pulmonary valve syndrome. As for babies with hypoplastic pulmonary arteries, the recommendation would continue to be that of reconstruction of the right ventricular outflow tract, leaving open the ventricular septal defect. The defect would then be closed surgically when the left and right pulmonary arteries had grown to an acceptable size. The recent introduction of balloon angioplasty provides yet another palliative option for young symptomatic infants presenting prior to the optimal age for primary repair. The procedure has been shown to promote growth of the ventriculo-pulmonary junction and pulmonary arteries, without affecting the incidence of requiring reconstruction of the right ventricular outflow tract. The technique, however, cannot be recommended without reservations since some patients continued to require treatment with propanolol for hypercyanotic spells after balloon dilatation.

\section{Outlet repair of tetralogy of Fallot \\ Hiromi Kurosawa \\ Jikei University, Cardiovascular Surgery, Tokyo}

The final goal subsequent to corrective cardiac surgery for tetralogy of Fallot is a low central venous pressure without production of conduction disturbances and with complete abolition of heart murmurs. The most characteristic hemodynamic feature of the lesion is a right-to-left ventricular shunt not only during systole, but also during the diastolic period of decreased pulmonary flow. This results in subnormal end-diastolic volumes of both ventricles. Any kind of surgical sequel or complication which increases ventricular volume load should, therefore, be avoided during surgery. Our preferred approach, the outlet repair, has four crucial features. First, we use the membranous flap and the posterior extension of the septomarginal trabeculation for closure of the ventricular septal defect. Second, we resect a sufficient, albeit limited, area of the outlet septum and the ventriculo-infundibular fold. Third, we insert a short patch in the outflow of the right ventricle occupying less than $30 \%$ of the right ventricular length. Fourth, we use a wide monocusp. Use of the membranous flap not only avoids complete heart block and residual leakage, but also avoids use of the septal leaflet of the tricuspid valve. Use of the latter structure courts the danger of increasing right ventricular end-diastolic pressure, and then increasing end-diastolic volume. The limited but ample resection of muscle within the subpulmonary area creates a wide infundibular channel, reduces the length of any needed outflow patch, and abolishes the heart murmurs. Use of a short patch also preserves good right ventricular contractility. A wide monocusp avoids pulmonary regurgitation and increases the volume load on the right ventricle. 'Outlet repair' of tetralogy of Fallot, therefore, produces a good quality of life with low central venous pressure, no conduction disturbances and no heart murmurs.

Unifocalization for tetralogy of Fallot with pulmonary atresia and systemic-to-pulmonary collateral arteries

Hiromi Kurosawa

Jikei University, Cardiovascular Surgery, Tokyo

Major systemic-to-pulmonary collateral arteries are encountered in tetralogy of Fallot with pulmonary atresia, with or without the presence of central intrapericardial pulmonary arteries. In the group co-existing with central pulmonary arteries, the goal of unifocalization is to incorporate the central pulmonary arteries. In those in which central pulmonary arteries are absent, unifocalization is to an artificially created central pulmonary artery, usually made from a roll of preserved pericardium. Most collateral arteries lack widespread intrapulmonary connections with other areas of the lung, so that 
arborization anomalies usually exist in their presence. Two-staged surgery is therefore necessary. Unifocalization is conducted first on one or both sides, followed by an intracardiac repair. This can take the form of a Rastelli type operation or reconstruction of the outflow tract using a patch. Since most collateral arteries originate from the descending aorta posterior to the hilums of the lung, unifocalization is usually achieved through a posterolateral thoracotomy in the fourth to the seventh intercostal spaces. The collateral arteries, together with the intraparenchymal pulmonary arteries, are well mobilized around the hilum. Nonstenotic collateral or intraparenchymal pulmonary arteries can be anastomosed directly to either native or artificially created central pulmonary arteries. If the sites chosen for anastomosis are far distant from each other, a graft of azygos vein or a small pericardial roll can be interposed. At the end of unifocalization, a modified Blalock-Taussig shunt is established to the central pulmonary arteries. To ensure long and adequate patency, unifocalization should be done without use of artificial grafts except in the creation of artificial central pulmonary arteries (if needed) and construction of the Blalock-Taussig shunt. Definitive intracardiac surgery is scheduled after a delay of several weeks or months. When bilateral artificial central pulmonary arteries have been created during bilateral unifocalization, these are connected by construction of another pericardial roll, either behind or in front of the ascending aorta. An extracardiac valved conduit is then constructed from the right ventricle to the confluence. In cases with native confluent central pulmonary arteries, the connection between the right ventricle and the pulmonary arteries is established using either an extracardiac conduit or an outflow parch.

\section{Diagnosis of complete transposition Gengi Satomi \\ Department of Pediatric Cardiology, The Heart Institute of Japan, Tokyo Women's Medical College, Tokyo}

Complete transposition (discordant ventriculo-arterial with concordant atrioventricular connections) is the most common cause of cyanosis occurring within the first year of life. Its prevalence is remarkably constant in the world, and is reported to occur once in every 4,300 to 4,500 live births. Complete transposition is particularly uncommon among premature infants and in those with low birth weights. The natural course is for $90 \%$ of the patients, if untreated, to die during the first year of life. Recent advances in cardiovascular surgery and interventional cardiology have modified markedly the management and long-term prognosis of this disease. The age at presentation is strongly related with the degree of cyanosis, which depends on the amount of mixing between the systemic and pulmonary circulations. Therefore, the patients with an intact ventricular septum and with poor interatrial mixing present earlier than do those with ventricular septal defect or with patency of the arterial duct.

Most of the patients are first noticed because of visible cyanosis. The diagnosis is confirmed by cross-sectional echocardiography, which demonstrates readily the parhognomonic combination of concordant atrioventricular and discordant ventriculoarterial connections. The pulmonary trunk is identified from the feature of its bifurcation into right and left branches in the parasternal shortaxis view. The pulmonary trunk arising from the morphologically left ventricle, and the aorta arising from the right ventricle, are clearly visualized in the subcostal coronal views in neonates and young infants. The state of the interatrial septum is best seen in subxiphoid short and long axis views. Bulging of the interatrial septum in these views suggests restriction of the interatrial communication. Pulsed Doppler techniques can be used for detecting the flow of blood across this communication. The pattern of shunting through the oval foramen as demonstrated by pulsed
Doppler echocardiography is indicative of whether or not shunting exists at other sites, such as a ventricular septal defect and/or a patent arterial duct. Color Doppler echocardiography is also helpful for detecting such defects when they are present. Coarctation or interruption of the aortic arch are frequent accompaniments of a ventricular septal defect associated with malalignment of the outlet septum, presumably because this arrangement obstructs the flow of blood from the right ventricle to the ascending aorta. Retrograde aortography is helpful for visualizing the morphology of the aortic arch more clearly. Diagnosis of the origin and distribution of the coronary arteries has become of increasing importance with the trend towards use of the arterial switch operation in recent years. Echocardiography is clearly useful in this respect, but may not show all branches of the coronary arteries nor their distributions. The "laid-back" aortogram, combined with use of a balloon catheter for aortic occlusion, has improved markedly the visualization of the coronary arterial trees.

Preoperative evaluation of patients considered for the Fontan procedure

Gengi Satomi

Department of Pediatric Cardiology, The Heart Instztute of Japan, Tokyo Women's Medical College, Tokyo

A number of physiological and anatomical features were proposed in 1978 by Choussat, Fontan and their colleagues as criterions for selection of patients for the Fontan procedure. As the number of successful cases of anastomotic atriopulmonary operations increased, the original ten criterions tended to be extended in one or more areas. This presentation will focus on the issue of current preoperative evaluation of indications for the Fontan operation. Later, the controversial sssue of extending the limits of these criterions will be briefly discussed.

Among the ten original features selected by Choussat et al, the most important remains the evaluation of the pulmonary circulation. Choussat et al suggested three features of the pulmonary circulation which should be followed, namely, mean pulmonary arterial pressure of less than $15 \mathrm{~mm} \mathrm{Hg}$, pulmonary resistance of less than 4 Wood unit ${ }^{2} \mathrm{~m}^{2} \mathrm{BSA}$, and ratio of pulmonary to aortic diameters of greater than 0.60. Subsequent experience has shown that presence of a mean pulmonary arterial pressure of less than $15 \mathrm{~mm} \mathrm{Hg}$ in isolation seems to be of relatively less importance. Rather, the important points concerning evaluation of the pulmonary circulation when determining indications for the Fontan procedure are the combination of, first, the size of the pulmonary arteries as a morphological index and, second, pulmonary resistance as a physiological index of the pulmonary arteries. Nakata's index, or the McGoon ratio, have been used to evaluate the size of the pulmonary arteries. A pulmonary arterial index of greater than 250 , which is $75 \%$ of the normal index, would be a suggested "cut-off" point based on the experience from Tokyo Women's Medical college. The pulmonary arterial resistance should be evaluated as a hemodynamic index by combining data of pressure and flow. There remain the problems in calculating the pulmonary arterial resistance, which will be discussed. Mayer and his co-workers have reported far better results in patients with pulmonary arterial resistance less than 2 Wood unit ${ }^{\cdot} \mathrm{m}^{2} \mathrm{BSA}$ than in those with resistances higher than 2 Wood unit $\mathrm{m}^{2}$ BSA (J Thorac Cardiovasc Surg 1986). We suggest, therefore, than an upper level of $2 \mathrm{Wood}$ unit ${ }^{\bullet} \mathrm{m}^{2} \mathrm{BSA}$ for pulmonary arterial resistance should be recommended as a safer criterion for the Fontan procedure. Limitations of age between 4 and 15 years old no longer appear to be an important issue with increasing experience in this type of operation. This is also borne out by the reports of successful modified Fontan procedures in patients with hypoplastic left heart syndrome by Ferrel et al (Circulation 1992), and from the 
experience with the modified Fontan operation in adults reported by Humes et al (Am J Cardiol 1988). Use of the Fontan repair with a fenestrated baffle has now further extended the limitations in its use.

\section{Pulmonary atresia with intact ventricular septum-surgical approach \\ C. $K . M o k$ \\ Grantham Hospital, Hong Kong}

Unless the arterial duct persists and adequately supports the pulmonary circulation and the obligatory right to left atrial shunt effectively decompresses the right heart, survival of babies born with pulmonary atresia and intact ventricular septum will depend on timely surgery tailored to the morphology and function of the right ventricle and tricuspid valve as well as associated significant coronary arterial anomalies. The goals of the surgical procedures are, first, to ensure adequate pulmonary blood flow; second, to decompress the right ventricle and promote forward flow to and growth of the pulmonary arteries; third, to minimize the deleterious effects, when present, of significant abnormal coronary circulation. It is generally accepted that maintaining ductal patency or construction of classical or modified central or peripheral shunts can all ensure adequate pulmonary blood flow. Transventricular or transarterial pulmonary valvotomy, along with patching of the right ventricular outflow tract, would decompress the right ventricle and promote its growth when there is an adequate subpulmonary infundibulum. Surgical excision and atrial septectomy, or thromboexclusion of the right ventricle, have been attempted to facilitate spontaneous closure of major fistulous communications with the coronary arteries. An effective way of managing the associated right ventricular-dependent coronary circulation, however, has yet to be found. Long term survivors are among those undergoing right ventricular outflow reconstruction or a modified Fontan repair. Because of the rarity and variability of expression of the anomaly, and in the absence of a multicentric prospective trial, the optimal surgical approach remains speculative. With the rapid advancement in technology and invasive cardiology, new strategies for management are imminent.

\section{Interventional cardiology in congenital heart disease}

\section{Michael Tynan}

United Medical \& Dental Schools of Guy's \& St Thomas's Hospitals, London

During the past decade, the practice of paediatric cardiology has undergone a profound change. It would not be too extravagant to say a revolution. This has been due to the wide acceptance of catheter interventions as alternatives, and adjuncts, to surgery. The surgical treatment of congenital heart defects is itself a relatively young discipline. In 1939, Gross and Hubbard reported the successful closure of the arterial duct whilst, in 1948, Brock published on the surgical treatment of pulmonary stenosis. By 1953, he had passed the 100 mark but it was in this year that the first reports of attempted catheter relief of pulmonary stenosis appeared from the Institute of Cardiology in Mexico City. The design of the catheter used in that centre prefigured that of the Park septostomy blade, but with the cutting element being a guide wire. It appears to have been effective but did not catch on. This was, perhaps, due to the primitive state of cardiac catheterization or to the power of the surgeon at that time. In 1966, Bill Rashkind really got the interventionists underway with the introduction of balloon atrial septostomy. This was a great advance when one remembers that, at that time, surgical septectomy had a mortality of 50 to 60 per cent in the early weeks of life. Over the succeeding decade and a half, furcher attempts were made to substiture catheter therapy for surgery. The most successful of these was plug closure of the arterial duct developed by Porstmann. This approach, however, required a large arterial introducer and was nor applicable to the paediatric age group. These forays into catheter intervention did not result in any widespread use of the methods until the rechniques of balloon angioplasty, used in the treatment of atherosclerotic disease, were applied to congenital cardiac malformations. To a great extent, this departure was initiated by the experimental work of Lock and his colleagues although it was Kan and White from Baltimore who first performed balloon dilatation in patients with pulmonary valvar stenosis. Today, the only indication for surgical pulmonary valvotomy is persistent stenosis after dilatation with a balloon of adequate size. Balloon dilatation has been extended to many other lesions.

In some, such as valvar aortic stenosis in childhood, intervention has also become the treatment of first choice. In others, intervention is widely used, but with some reservations. Aortic coarctation is one such lesion. In yet others, the efficacy is in dispute, for example subvalvar aortic stenosis. There are other lesions for which intervention is ineffective, such as balloon dilatation of pulmonary venous stenosis. Finally, there is a group of applications which can be described as 'under evaluation' or even as experimental. In this last category are procedures with an ambiguous status. For example, catheter closure of the arterial duct is considered the treatment of choice in most centres in Europe but is still limited by law to investigational status in the United States of America, the country of its origin.

Intervention has had a marked effect on the practice of paediatric cardiology. In our own hospital, intervention now accounts for half the catheterizations performed on infants whereas, ten years ago, only one-tenth of such catheterizations were performed for interventional purposes. There is, therefore, a major role for interventional techniques in treatment of structural congenital heart anomalies. Advances continue making therapy more effective, more 'patient friendly' and more cost-effective.

Visualization of the pulmonary arterial tree in the tetralogy of Fallot with pulmonary atresia

Michael Tynan

Guy's Hospital, London

The anatomy of the pulmonary arterial tree in the tetralogy of Fallot with pulmonary atresia is complex. The objectives of imaging are multiple. The presence, size and anatomy of the central, usually referred to as "true", pulmonary arteries must be defined. The presence, or absence, of an arterial duct, or ducts, must be established and the presence, or absence, of aorto-pulmonary collateral arteries must also be defined. All methods of imaging have their place in the study of this condition. Echocardiography may well demonstrate confluent central pulmonary arteries, especially when the pulmonary arterial blood supply is unifocal and derives from one arterial duct. When the pulmonary arteries are small, however, echocardiography may be unhelpful in this regard. In any event, the visualization of pulmonary arteries out into the lung substance is necessary and, thus, echocardiography has only a limited role. The mainstay of investigation has always been angiocardiography. In the presence of an arterial duct, good visualization of the pulmonary arteries can be obtained either from an aortogram or from an injection into the pulmonary arteries having traversed the duct with the catheter. Aortography will also demonstrate the number and sites of origin of major aortopulmonary collateral arteries. On occasion, however, particularly when the arterial duct or ducts are closed, aortography alone may not demonstrate the central pulmonary arteries, even though they are present. This may well be due to some overlap of collaterals and "true" pulmonary arteries on the normal anterior or posterior projection of the aortogram. To minimize this possibility for confusion, the aortogram should be performed with 45 head up tilt. Then the pulmonary arteries will be seen towards the lower part 
of the frame whilst the collateral arteries will tend to be in the upper part of the frame.

Since a collateral artery may branch and supply both lungs, the mere presence of a bifurcating artery does not mean that these are "true" pulmonary arteries. Even if this manoeuvre has failed to demonstrate the pulmonary arteries, then direct injections into the collateral arteries may well give good visualization of the central pulmonary arteries, due to the fact that one or all of them will anastomose with the pulmonary arteries. Should this method fail to demonstrate central pulmonary arteries, then a pulmonary venous wedge angiogram should be performed.

Today, a further adjunct to the demonstration of the anatomy of the pulmonary tree is magnetic resonance scanning. This is particularly useful in the demonstration of central pulmonary arteries. It has some limitations when it comes to visualizing the collaterals since, although their origin can be seen, their tortuosity makes tracing their course usually somewhat difficult.

It may be that full visualization of the anatomy and the segmental distribution of the pulmonary blood supply from the central pulmonary arteries and the collaterals has to await palliative surgery - either construction of a Blalock Taussig anastomosis or the establishment of continuity from the right ventricle to the pulmonary arteries, for it is not only the presence or even just the distribution of the central pulmonary arteries that is important. Since stenoses are not infrequent, be they congenital or acquired after surgical palliation, they must be sought, defined, and, if necessary, treated.

\section{Diagnosis of valvar diseases in children the role of transesophageal echocardiography \\ Maurice Leung \\ Grantham Hospital, Hong Kong}

Children and young adults suffering from valvar diseases usually have typical physical and auscultatory findings. Confirmation of their diagnosis and evaluation of the severity of the lesions have, in the past, largely depended on cardiac catheterisation and angiography. Recently, transthoracic echocardiography has provided a noninvasive and yet definitive diagnosis for these valvar abnormalities. The ultrasonic visualization of the heart from the oesophagus (transesophageal echocardiography) has the added advantage of being in close proximity to the posterior cardiac structures, thereby allowing a clearer definition of the valvar morphology. From January 1991 to August 1992, we used biplane transesophageal echocardiography to study 30 children and young adults with predominant cardiac valvar diseases. Their ages ranged from 2.5 to 20 years (mean of 12.5 years) and body weight from 12 to $67 \mathrm{~kg}$ (mean of $36.2 \mathrm{kgs}$ ). A total of 33 procedures were performed, 8 of which were under general anaesthesia (3 each during open-heart surgery and interventional catheterisation, 2 during diagnostic catheterisation). The other 25 procedures were performed with the patient receiving local anaesthetic spray and an intravenous sedation. All children received transthoracic ultrasonic examination prior to the transesophageal echocardiography, and 20 patients had additional cineangiography for comparison. Of the 30 children, 13 suffered from predominant mitral valvar disease, 15 had predominant aortic valvar lesions and 2 had mixed aortic and mitral valvar pathologies. When compared with the transthoracic echocardiographic findings, additional information on the diagnosis relating to the precise pathology could be gained from transesophageal echocardiography in 19 patients (63\%). Transesophageal echocardiography was particularly useful in the peri-operative assessment and diagnosis of residual lesions in 3 patients undergoing open-heart surgery ( 2 of cleft mitral valve, 1 of valvar replacement at both the aortic and mitral regions), and in 3 patients undergoing interventional catheterisation ( 2 of balloon dilatation of the aortic valve and 1 of mitral valve). Hence, transesophageal echocardiography directly contributed to the better management of 9 patients $(30 \%)$. One patient (3\%) with significant residual supravalvar aortic narrowing following surgical correction was missed by both methods of echocardiography but clearly shown by angiography. There were no major complications arising from the transesophageal procedures. We conclude that transesophageal echocardiography contributed significantly to the diagnosis and management of children suffering from cardiac valvar disease.

\section{Complete transposition-long-term follow-up after atrial redirection operation \\ J. R. Zuberbuhler \\ Children's Hospital of Pittsburgh, Pittsburgh}

Surgical repair of complete transposition (concordant atrioventricular and discordant ventriculo-arterial connections) is now almost exclusively by the "arterial switch" procedure. There is consensus that this operation should afford better long term results than either the Mustard or the Senning atrial redirection procedures. In an effort to provide a baseline for comparison, we have examined our experience with the atrial redirection operations. We report the medium term follow-up of 95 patients who survived a Mustard procedure, and 45 who survived a Senning operation. Problems reported in survivors of these procedures include right ventricular and tricuspid valvar dysfunction, baffle leak, systemic and pulmonary venous obstruction, arrhythmias, diminished exercise capacity and sudden unexpected death.

Right ventricular dysfunction has been attributed either to persisting high right ventricular afterload or to chronic myocardial hypoxia prior to repair. Since right ventricular function may be well maintained for decades in individuals with congenitally corrected transposition, and since a recent study showed a much lower incidence of right ventricular dysfunction in patients who had their operation very early in life, the latter may be the more important factor. In our series, right ventricular ejection fraction by MUGA without exercise was abnormal in $36 \%$ of asymptomatic patients following the Mustard operation, and by exercise MUGA was abnormal in $63 \%$. Resting left ventricular ejection fraction was abnormal in $10 \%$, and was abnormal in $40 \%$ following exercise. Hemodynamically significant tricuspid regurgitation was very rare in our experience, occurring in only 1 of 140 patients. Valvar replacement was successful in this case, and the patient is doing well 16 years later.

Estimates of the frequency of baffle leak range from $25 \%$ to $90 \%$ in the literature. In our series, a leak was noted in $25 \%$ of the 82 patients who underwent cardiac catheterization following the Mustard procedure. Transesophageal echocardiography has proven to be a very good technique for identifying baffle leak, and is much superior to transthoracic echo.

Systemic venous obstruction in our series occurred in 13\% of patients undergoing a Mustard operation and in $4 \%$ of those undergoing a Senning procedure. The obstruction was sometimes clinically silent, and here also transesophageal echo is far superior diagnostically to transthoracic echo. Operation was required in 6 of the 11 patients in our series with systemic venous obstruction. Pulmonary venous obstruction was seen only early in our series of Mustard operations, and usually required reoperation. Pulmonary venous obstruction, however, occurred in 9 of 45 survivors of the Senning operation (20\%). Pulmonary venous obstruction should be suspected either if pulmonary venous markings are increased on chest radiograph or if a faint continuous murmur is present. Diagnosis is by either transthoracic or transesophageal echocardiography. In all, a total of 15 of 140 survivors required reoperation $(11 \%)$. 
The frequency of arrhythmia depends upon how diligently it is searched for. In our series, only $50 \%$ of patients had normal sinus rhythm on all surface electrocardiograms, and only $30 \%$ had normal sinus rhythm on all testing modalities including Holter monitoring and exercise testing. Sick sinus syndrome is the most common arrhythmia and the frequency increases with the interval since operation. Ventricular and atrial ectopy are common, and atrial flutter is sometimes poorly tolerated and is a marker for sudden death. Reports of 10 year actuarial survival rates in the literature range from $75-90 \%$. Late death in our series occurred in 14 of 95 early survivors of the Mustard operation (15\%) and in 4 of the 45 early survivors of the Senning operation (9\%). The most common cause of late death was arrhythmia.

\section{Tetralogy of Fallot-diagnostic features \\ J. R. Zuberbubler}

\section{Children's Hospital of Pittsburgh, Pittsburgh}

The diagnosis of tetralogy of Fallot is usually not difficult. Most individuals with this anomaly present with cyanosis and a loud left sternal border systolic murmur, but there are exceptions at both ends of the spectrum of severity. The two hemodynamıcally important parts of the tetralogy are the large unrestrictive ventricular septal defect and the muscular obstruction within the right ventricular outflow tract, the latter being the morphologic variable that dictates the corresponding clinical spectrum. If pulmonary stenosis is relatively mild, there will be no right-to-left shunt and, therefore, no cyanosis. The murmur will be loud and long, and occasionally pulmonary arterial pressure is sufficiently high to generate an audible pulmonary closure sound and, therefore, a split second heart sound. With very severe pulmonary stenosis, there will be a large right-to-left shunt and severe cyanosis. The closure sound of the pulmonary valve is never audible and the second heart sound is always single. This second heart sound is usually loud at the "pulmonary" area, but represents closure of the aortic rather than the pulmonary valve. The systolic murmur becomes softer and shorter with increasing severity of pulmonary stenosis, since less blood traverses the right ventricular outflow tract. A transient increase in pulmonary stenosis accounts for the softening or disappearance of the systolic murmur during a hypercyanotic spell. An aortic ejection sound (not a "click") heard best at the apex and sounding like a split first heart sound is common with very severe tetralogy of Fallor, especially when there is pulmonary atresia. A pulmonary ejection click is very uncommon and gallop sounds are not part of the auscultatory spectrum of tetralogy of Fallot. The diagnosis is made by demonstrating the characteristic anatomic features by echocardiography. Coronary arterial anomalies, which are not rare, and distal abnormalities of the pulmonary arteries, including hypoplasia, stenosis and arborization abnormalities, are much better demonstrated by angiography. In some institutions, cardiac catheterization is done routinely prior to surgical repair. Right ventricular hypertrophy is the rule on the electrocardiogram. The heart is never enlarged on chest $\mathrm{x}$-ray in uncomplicated tetralogy of Fallot. The differential diagnosis includes an anomalous right ventricular muscle bundle in the presence of a ventricular septal defect, a doubly committed subarterial ventricular septal defect with pulmonary stenosis, double outlet right ventricle with bilateral infundibulum and pulmonary stenosis, and atrioventricular septal defect with pulmonary stenosis. Each can be diagnosed with proper imaging, either with echocardiography or cineangiography.

\section{Infective endocarditis}

J. R. Zuberbubler

Children's Hospital of Pittsburgh, Pittsburgh

The 30 year experience with infective endocarditis at the Children's
Hospital of Pittsburgh has been reviewed, and a total of 94 patients up to 21 years of age have been identified. The number of cases, clinical findings, cardiac substrate, infective organism, frequency of cardiovascular damage and need for surgical intervention, and mortality rates were determined in each decade and trends noted. These trends were compared and contrasted to those reported in the recent literature. "Classic" findings (such as Janeway lesions, Osler's nodes, Roth spots and splinter hemorrhages) were rare throughout. The most common clinical findings in each decade included fever, increased sedimentation rate and evidence of pre-existing heart disease. Infectious endocarditis is reported in the literature to be increasing in frequency. Our findings are in agreement, since the number of cases increased from 14 to 28 to 52 cases in the three decades, respectively. The cardiac substrate has been reported to be changing, with diminishing frequency of rheumatic heart disease and increasing frequency of prolapse of the leaflets of the mitral valve. Rheumatic heart disease was present in only 4 of the 94 cases in our series, and prolapsed mitral valve in only 2 . In our series, simple obstructive anomalies were present in $18 \%$, simple shunts or valvar regurgitation in $26 \%$, complex (usually cyanotic) malformations in $46 \%$, and no heart disease in $10 \%$. Staphylococci, and "unusual" organisms, are supposedly becoming more common. But, in the three decades of our study, the relative frequency of streptococci and staphylococci was unchanged and either streptococci or staphylococci were the infective organisms in $93 \%, 75 \%$ and $89 \%$ of the children in the three decades, respectively. The frequency of "unusual" organisms declined from $14 \%$ to $8 \%$ in the last two decades.

Cardiac damage occurred in about $30 \%$ of cases in each decade. The aortic valve was the structure most frequently involved. Surgery was necessary in 17 of the $28(61 \%)$ with damage and 5 patients died at operation. A total of 16 patients died. Other causes of death included congestive heart failure, rupture of the aorta or pulmonary trunk, persistent infection, and systemic or pulmonary embolization. The death rate has been reported to be higher in surgically treated cases of congenital heart disease, but we could not confirm this finding. The mortality rate is reportedly declining and our study confirmed this, the mortality rate in the three decades being $36 \%$, $25 \%$ and $8 \%$, respectively.

\section{Anatomic features of pulmonary atresia with intact ventricular} septum

\section{J. R. Zuberbubler}

Children's Hospital of Pittsburgh, Pittsburgh

Pulmonary atresia with intact ventricular septum is a complex congenital cardiac malformation. At one time, the major focus was on the right ventricle, and it was thought that the size of this chamber largely dictated the choice of surgical procedure and also determined long-term prognosis. This was an overly simplistic view, and it is now realized that this malformation is more than just complete right ventricular outflow tract obstruction with a smallish right ventricle.

Right ventricular size is one of the major variables and constitutes a spectrum from tiny to much larger than normal. The hypoplastic right ventricle seen in most cases is not just a miniature but normally formed structure. The three parts of the right ventricle may be affected in different degrees. The inlet portion is always preserved but the outflow tract and apical trabecular portion may be small or even absent. The form of the right ventricle is predictive of other features of this malformation.

The size of the tricuspid "annulus" correlates strongly with the size of the right ventricle but the degree of dysplasia of the valve is probably inversely correlated. A thin, well formed tricuspid valve is present in some very dysplastic right ventricles, and the largest right 
ventricles are found in hearts with very dysplastic tricuspıd valves, the valve having features of Ebstein's anomaly. The presence of dysplasia and the consequent tricuspid regurgitation may actually be a major determinant of right ventricular growth.

The arrangement of the pulmonary valve also correlates with right ventricular size and morphology. The valve has a central smooth area without raphes if the right ventricular infundibulum is only mildly or moderately narrow, while central raphes are found in hearts with atretic or severely stenotic infundibulums in which muscle interposes between the cavities of right ventricle and pulmonary trunk. The pulmonary trunk itself is usually of good size, but the size does not correlate with that of the right ventricle. Pulmonary blood flow is almost always by way of a patent arterial duct, with systemic-arterial collaterals being exceedingly rare.

Coronary arterial anomalies are common in hearts with very hypoplastic right ventricles. Fistulous communications with the right ventricle, and coronary arterial stenoses are both seen and coronary flow may be compromised if right ventricular pressure is lowered following surgical relief of obstruccion of the right ventricular outflow tract. This occurs if there is major obstruction proximal to important branches supplying the myocardium. The potential impact on the myocardium is tempered by collateral arteries, and an obstruction in one coronary artery is probably not a contraindication to right ventricular decompression.

\section{The clinical examination, a dying art?}

\section{J. R. Zuberbubler}

Children's Hospital of Pittsburgh, Pittsburgh

The physical examination is a very important part of clinical care in the child with suspected or proven heart disease, providing the examination is carefully and expertly done. There is a regrettable tendency to substitute expensive "high technology" for a clinical evaluation, even in situations where the clinical examination is likely to be equally or even more effective. Areas in which the clinical examınation is potentially useful include screening, specific diagnosis and quantitation of the severity of hemodynamic abnormalities. The physical examination is the best way of screening for the presence of heart disease. It is certainly the most cost-effective method, but also is the most effective in general. It is, indeed, the way in which most heart disease is first diagnosed in childhood. Consider that congenital heart disease in the pediatric age range almost always presents as cyanosis, congestive heart failure, or a murmur in an otherwise well child. In each case, the physical examination is paramount in diagnosis.

Many, if not a majority of acyanotic anomalies, can be specifically diagnosed by a physical examination. This can be illustrated by considering several classes of congenital heart disease in turn.

A triad of findings is present in $90 \%$ of children comıng to operation for atrial septal defect. This triad includes a soft basal systolic ejection murmur, a soft low left sternal border mediumpitched mid-diastolic murmur (not a rumble!!) and fixed splitting of the second heart second. The triad is almost never found in the absence of a hemodynamically significant left-to-right shunt at the atrial level.

A loud low left sternal border systolic murmur most likely represents a ventricular septal defect. Such a murmur when soft is likely to be either a small ventricular septal defect or a functional murmur. The latter is identified by its "vibratory" quality and is easily distinguished from the murmur of a ventricular septal defect. The caveat that there is no "typical" murmur of a ventricular septal defect must be noted. The murmur may be loud or soft. It is not always plateau and pansystolic. Very small ventricular septal defects often produce a short early systolic murmur, and the murmur of a ventricular septal defect partially obstructed by tissue tags derived from the tricuspid valve ("aneurysm of the membranous septum") is usually crescendo. The murmur of a doubly committed subarterial ventricular septal defect is usually at the high, not the low, left sternal border, and is usually crescendo-decrescendo. A large flow ventricular septal defect produces a soft diastolic murmur of relative mitral stenosis at the apex.

A continuous murmur, loudest at the high left sternal border, is almost always due to patency of the arterial duct. A jugular venous hum always disappears in the recumbent position and should, therefore, never be confused with a continuous murmur from a duct.

Typical findings of valvar pulmonary stenosis include a high left sternal border systolic ejection murmur, which is typically short if obstruction is mild and long if severe. The pulmonary component of the second heart sound is soft if stenosis is severe and pulmonary arterial pressure, therefore, low. A widely split second heart sound is usually present if stenosis is mild. A high-pitched early systolic ejection sound is heard at the high left sternal border and becomes softer with inspiration.

In valvar aortic stenosis, the systolic ejection murmur is loudest at the high left sternal border and radiates to the neck. there is poor correlation of loudness and length of the murmur with severity of the obstruction, although important obstruction is very rare in the absence of a thrill (does not apply to neonates with critical aortic stenosis). An early systolic ejection sound (not high-pitched and "clicky") is present at the apex.

The diagnosis of aortic coarctation is made by finding weaker pulses and lower blood pressure in the legs than in the right arm. (The left subclavian artery may be involved in the coarctation and pulses and blood pressure may be equal in the legs and the left arm.)

The classic murmur of aortic regurgitation is high-pitched, decrescendo, and loudest at the mid and low left sternal border. The murmur may be soft, and is best heard with the patient squatting. The physical examination is the best non-invasive way to quantitate the regurgitation (better than echo/Doppler at present). Severe aortic regurgitation is evidenced by full pulses, a wide pulse pressure, an Austin Flint murmur (diastolic rumble at the apex) and by a Duroziez' sign (to and from murmur if the femoral artery is compressed by the stethoscope head, tilted so that the distal edge compresses the femoral artery-retrograde flow during diastole causes the diastolic component of the to and fro murmur).

The quintessential finding of mitral regurgitation is a systolic murmur loudest at the apex. The only other anomaly that can cause this finding is an apical muscular ventricular septal defect. Mitral regurgitation cannot be quantitated by the systolic murmur alone, but severe regurgitation always produces an active apical impulse and an apical mid-dlastolic rumble of relative mitral stenosis.

In summary, physical examination and echo/Doppler techniques are complementary rather than competing modalities. The physical examination is the better screening tool, and in some cases the better means for quantitating severity. Echo/Doppler technology is very useful for confirmıng diagnoses suspected on physical examination, and in many cases (such as obstruction) is very effective in quantitating severity.

Left ventricular function after palliative for pulmonary atresia with intact ventricular septum

R. Calabro, G. Pacileo, C. Pisacane, M.G. Russo, C. Vosa

University of Naples, Naples

The left ventricle in patients with pulmonary atresia with intact ventricular septum, in consequence of its receipt of both systemic and pulmonary venous flows, is exposed to volume overload. Such work is increased still further by the construction of palliative systemic-topulmonary arterial shunts. It may well be that this additional load, when superimposed on progressive coronary arterial and myocardial 
abnormalities, may produce impaired diastolic and systolic function of the left ventricle even in the absence of fistulous communications from the right ventricle to the coronary arteries. To investigate this possibility, westudied parameters of systolic and diastolicleft ventricular function by cross-sectional and Doppler echocardiography in 14 patients (aged from 15 days to 16.5 months; mean age $4.03 \pm 5.6$ months) with pulmonary atresia and intact ventricular septum in the absence of fistulous communications who had undergone construction of a systemic-to-pulmonary arterial shunt. The findings were compared with a group of eight control subjects matched for age, body surface area and heart rate. Follow-up after palliation ranged from 12 days to 16.3 months, with a mean follow-up of $3.67 \pm 5.6$ months. Left ventricular ejection fraction was decreased $(61 \pm 7 \%$ vs $68 \pm 5 \%$; $=$ $0.22)$ while the index of end-diastolic volume was increased $(72.7 \pm$ $10.8 \mathrm{ml} / \mathrm{m}^{2} ; \mathrm{p}=0.0001$ ) compared to the controls. The left ventricular mass indexed for body surface area, however, was normal (67.88 \pm $20.2 \mathrm{~g} / \mathrm{m}^{2}$ vs $\left.76 \pm 10 \mathrm{~g} / \mathrm{m}^{2} ; \mathrm{p}=\mathrm{NS}\right)$. The index of left ventricular shape was increased in all the shunted patients $(1.27 \pm 0.26 \mathrm{vs} 1 \pm 0.01 ; \mathrm{p}=$ $0.009)$. A significant inverse correlation was found between the ejection fraction and time of follow-up $(r=-0.71 ; p=0.04)$ as well as with the shape index $(r=-0.76 ; p=0.048)$. An increased $E / A$ ratio was also found in the shunted patients $(0.65 \pm 0.16 \mathrm{vs} 1.35 \pm 0.90 ; p=$ 0.009 ) as well as a decrease in the normalized peak rate of filling (4.16 \pm 0.13 vs $6.88 \pm 0.68 ; p=0.0001)$, in the increased peak velocity of the $A$ wave $(0.95 \pm 0.17 \mathrm{~m} / \mathrm{s}$ vs $0.51 \pm 0.16 \mathrm{~m} / \mathrm{s} ; \mathrm{p}=0.0001)$ and a prolongation of the isovolumic time of relaxation $(46 \pm 5.4 \mathrm{~ms}$ vs 34 $\pm 6.2 \mathrm{~ms} ; \mathrm{p}=0.0001)$ and of the time of deceleration $(196.4 \pm 32.2 \mathrm{~ms}$ vs $116 \pm 21.4 \mathrm{~ms} ; \mathbf{p}=0.0001$ ). A good correlation was found between the normalized peak rate of filling and follow-up $(r=-0.80 ; p=0.04)$. No differences were found in the velocity curves as estimated by Doppler for pulmonary venous flow between the groups. Our data show that, with time, there is an increasing compromise of systolic and diastolic function in the left ventricles of patients undergoing palliative shunting for pulmonary atresia with intact ventricular septum in the absence of fistulous communications from the right ventricle to the coronary arteries. Reconstruction of the right ventricular outflow tract is, therefore, to be recommended as soon after palliation as is possible.

\section{Adolescent cardiology}

John E. Deanfield

Hospital for Sick Children, London

The outlook for patients with congenital heart disease has been transformed by improvements in medical and surgical treatment so that the majority of children, even with complex defects, now survive into adolescence and adult life. This success story has radically increased the size and complexity of the population of young adults with congenital heart disease, and this population will continue to grow. Such patients require specialized services, which in most countries have not been developed. As a result, patients are often managed by adult physicians who are unfamiliar with the anatomy and physiology of complex congenital heart defects. Total correction in childhood is not the rule and many patients need reoperation. These patients include those needing reoperation after definitive repair, for example, those with extracardiac conduits, residual and new defects, staged repair for complex defects, and heart and lung transplantation. Other adults may require their first operation for congenital heart lesions that were well tolerated during childhood as, for example, Ebstein's malformation. Medical problems in natural survivors and postoperative patients include arrhythmia, deteriorating hemodynamics, endocarditis and management of non-cardiac problems secondary to cyanosis. In addition, psychosocial problems such as employment, life and health insurance, sports, sexual activity, contraception and pregnancy are of great importance to these individuals.
Long-term follow-up data is crucial as a feedback, so that initial strategies of management in childhood can be refined. Late follow-up data have already changed the surgical approach in childhood for common defects such as complete transposition. This supports the centralization of medical expertise to accumulate knowledge about the fate of the group as a whole. This will enable training of the necessary group of cardiologists, surgeons and allied health professionals as well as facilitating research. The new population of adults with congenital heart disease requires considerable investment by the medical community to match the standards of care set in childhood.

\section{Tetralogy of Fallot-long-term follow-up} John E. Deanfield

\section{Hospital for Sick Children, London}

The management of tetralogy of Fallot has been one of the success stories of congenital heart disease. The natural history of this defect is poor, with only $25 \%$ of patients alive at 10 years, $11 \%$ at 20 years and $3 \%$ at 40 years. In the developed world, the majority of patients have undergone palliative surgery or more usually, repair in childhood. Late survival is excellent, even in patients who underwent repair during the very early years of open heart surgery. At the Mayo Clinic, the cumulative 30 year survival in patients undergoing successful surgery from $1956-1960$ was $86 \%$ compared to $95 \%$ in age and sexmatched controls. Surgery, however, cannot be considered "curative". Survival, even in the best series, is slightly but significantly worse than for the control population. Risk factors for adverse late outcome include older age at surgery, preoperative congestive cardiac failure, previous construction of a Potts shunt, persisten right ventricular hypertension, and residual ventricular septal defects. Late death may be sudden, due to ventricular tachycardia/fibrillation or, very rarely in the current era, due to conduction damage and complete heart block. The optimal management to reduce the risk of sudden death is still controversial.

The late functional outcome is excellent in the majority of patients. Most lead normal lives but results appear to be better when repair is performed at a younger age. Exercise capacity is essentially normal in children repaired before the age of 5 years. Persistent or recurrent symptoms are usually the result of incomplete relief from right ventricular systolic hypertension or recurrent or residual ventricular septal defects. These problems are often manifested early after surgery and may require reoperation. Pulmonary regurgitation is likely to be an increasing problem with very long-term follow-up. It may cause cardiomegaly with decreased exercise capacity. Insertion of a pulmonary homograft may be required but the precise indications and timing remain unclear.

Patients subsequent to repair of tetralogy of Fallot should be encouraged to lead a normal life without restriction unless there is a residual electrophysiological or hemodynamic abnormality. They should, however, remain under long-term medical follow-up.

\section{Ebstein's malformation-natural history \\ John E. Deanfield}

Hospital for Sick Children, London

The severity of Ebstein's malformation of the tricuspid valve is variable and accounts for the wide clinical spectrum, from fetal and neonatal death to survival up to the 8 th decade of life. The diagnosis is now much easier in the era of cross-sectional echocardiography and this has altered the understanding of the natural history. We have reviewed 220 cases: 21 presenting in fetal life, 88 as neonates, 23 as infants ( 1 month-2 years), 65 as children (2-18 years) and 23 as adults (18-71 years) over a 3 month to 30 year (mean, 8 year) follow-up. The most common mode of presentation was an abnormal routine prenatal scan for the fetuses $(86 \%)$, cyanosis for neonates $(73 \%)$, heart failure for infants $(40 \%)$, incidental murmur for 
children (66\%), and arrhythmia for adults (50\%). The earlier the presentation, the higher the incidence of associated cardiac defects, the most common being pulmonary stenosis or atresia (fetal $52 \%$, neonatal $49 \%$, infants $48 \%$, children $17 \%$, adults $4 \%$, p $>0.05$ ). In contrast, pre-excitation or arrhythmia were more frequent in those presenting later in life (fetal $10 \%$, neonatal $10 \%$, infants $17 \%$, children $35 \%$, adults $65 \%, \mathrm{p}<0.05$ ).

Outcome was poor in the fetus and neonate. Of 21 fetuses, there were 8 terminations, 4 intra-uterine and 6 post-natal deaths and 3 survivors, (1 year survival 19\%). Of 88 neonates, 32 died during follow-up ( 5 year survival $65 \%$ ). Deaths were mainly due to hemodynamic deterioration $(17 / 32,53 \%)$, was perioperative $(9 /$ $32,28 \%)$ or occurred late and suddenly $(5 / 32,16 \%)$. Outcome was best predicted by an echocardiographic grade of severity. Older age at presentation was associated with a better prognosis ( 5 year survival of infants $78 \%$, children $95 \%$ and adults $86 \%$ ). Ebstein's malformation is compatible with prolonged survival into adult life with few problems. Symptoms and signs, when they develop, are cyanosis due to right-to-left shunt at atrial level, dyspnoea secondary to hypoxia, and recurrent supraventricular arrhythmia. Ebstein's malformation is often associated with ventricular pre-excitation which may involve one, or more, usually right-sided accessory pathways. Progressive heart failure may develop and be related to left-sided abnormalities (such as excessive myocardial fibrosis) as well as right-sided abnormalities. In patients with increasing cyanosis, congestive cardiac failure or uncontrolled arrhythmia, and in those in New York Heart Association class III or IV, outcome is poor and surgery should be undertaken. This was performed in 79/220 patients (palliation in 32, tricuspid valvar surgery in 30 , and other open heart procedures in 17). Perioperative mortality for tricuspid valvar surgery was $5 / 5(100 \%)$ in children less than 2 years of age at operation, and $1 / 5(20 \%)$ in those over 2 years $(\mathrm{p}<0.05)$.

In Ebstein's malformation, the earlier the presentation the worse the prognosis, largely due to hemodynamic problems and poor surgical outcome in neonates and infants. In children and adults with Ebstein's malformation, incidental finding or arrhythmia are most common at presentation and the long-term outcome is superior.

Physiology of Fontan circulation

John E. Deanfield

Hospital for Sick Children, London

The Fontan operation, or one of its several modifications, is being performed as the definitive repair for an increasingly wide range of congenital heart abnormalities in which a biventricular circulation cannot be achieved. These include tricuspid atresia, mitral atresia, double inlet ventricle, hearts with one hypoplastic ventricle, straddling atrioventricular valves and Ebstein's malformation. If there is no ventricle between the right atrium and the pulmonary arteries (atrial dependent circulation), the right atrial pressure must be higher than the left atrial pressure by an amount which is related to the resistance to right atrial outflow (in practice pulmonary vascular resistance plus any resistance imposed by the connection between the venous return and the pulmonary arteries). This imposes constraints on the circulation which were expressed by Choussat's 10 commandments. Since then, experience has shown that, of these, only the pulmonary vascular resistance, ventricular function and atrioventricular valvar competence are critical. Recent reports, nonetheless, have emphasized that deviations from several criterions are associated with an increased long-term risk and worse functional result.

The desirability of incorporating the right atrium into the Fontan circuit has been questioned. In conditions other than tricuspid atresia, the thin-walled right atrium is unlikely to contribute energy. Hydrodynamic research has suggested that a valveless chamber may generate turbulence and increased resistance to forward flow.
Furthermore, a hypertensive right atrium may be responsible for supraventricular arrhythmia, particularly atrial flutter. This problem has been shown to increase with follow-up after surgery and may be very poorly tolerated in the Fontan circulation. As a result, the Fontan circuit has been modified to exclude the right atrium by a "total cavopulmonary connection" (end-to-side anastomosis of the superior caval vein to the undivided right pulmonary artery, a bidirectional Glenn shunt, and construction of a composite intraatrial tunnel using the posterior wall of the right atrium). Ongoing follow-up with postoperative electrophysiological and hemodynamic studies will indicate whether this procedure has advantages over the classical atriopulmonary connection.

All circuits for the Fontan procedure and its modifications should currently be regarded as definitive palliation rather than as repair or cure since the long-term consequences of elevated systemic venous pressure; minimally pulsatile pulmonary flow, and altered filling characteristics of the ventricle are unknown. Recent evidence has suggested that there is an increasing hazard with time even after the so-called "perfect Fontan", with no obvious risk factors other than the Fontan circulation itself.

\section{Extracorporeal membrane oxygenation for pediatric cardiac} support

Ralph D. Siewers and Pedro J. del Nido Children's Hospital of Pittsburgh, Pittsburgh

Extracorporeal membrane oxygenation has become an accepted mode of therapy for severe neonatal and pediatric respiratory insufficiency which is inadequately responsive to more conventional forms of treatment. Because extracorporeal oxygenation essentially provides mechanical "right heart bypass", its use for circulatory support in children has been met with increasing enthusiasm. At Children's Hospital of Pittsburgh since 1981, we have used membrane oxygenation in over 500 cases of various forms of respiratory insufficiency. Our experience specifically for hemodynamic support is smaller, numbering 45 cases from 1981 through June, 1992. In the group of parients placed on support following corrective operations for congenital heart disease, the failures have most often been associated with failure of multiple organ systems, specifically damage to the central nervous system caused by inadequate cardiac output and/or hypoxia prior to initiation of mechanical support.

The cardiomyophathy/myocarditis group is more complex. Most of these patients with impairment of cardiac function severe enough to require mechanical support have required cardiac transplantation. One of our patients with acute myocarditis recovered without transplantation after 7 days of support. Of the 10 patients in which membrane oxygenation was used as a bridge to transplant, 8 received a transplant, one developed sepsis precluding surgery and one manifested problems in the central nervous system related to the period prior to institution of extracorporeal oxygenation. Treatment was terminated.

Five patients with congenital cardiac anomalies have been placed on membrane oxygenation prior to operative intervention. Three with torally anomalous pulmonary venous connection had respiratory failure secondary to pulmonary edema and underwent successful correction following 1-4 days of support. One developed profound left heart dysfunction following embolic infarction of left atrial thrombus. Emergent circulatory support was successful but discontinued when massive injury was found in the central nervous system also due to emboli. The other patient in this group had complex cyanotic heart disease and suffered an arrhythmic arrest (unobserved) prior to corrective surgery. Oxygenation was used to support cardiopulmonary resuscitation but was discontinued when failure became clear in the central nervous and other systems.

We conclude that rescue of children with post-cardiotomy cardiac 
failure became clear in the central nervous and other systems.

We conclude that rescue of children with post-cardiotomy cardiac arrest by extracorporeal oxygenation is feasible in selected patients, even after prolonged cardiopulmonary resuscitation providing the period prior to resuscitation was observed and short. Extracorporeal oxygenation is a reasonable bridge to transplantation in children although left heart decompression may be necessary. Earlier intervention with extracorporeal support has reduced the incidence of multisystem organ failure in post-cardiotomy patients with severe cardiac dysfunction.

Tetralogy of Fallot with pulmonary atresia-long-term follow-up John E. Deanfield

Hospital for Sick Children, London

A number of surgical options are now employed for the treatment of patients with tetralogy of Fallot with pulmonary atresia. Understanding of the natural history and outcome of the interventional strategies is complicated by the biased spectrum of patients usually reported. Of 218 patients with tetralogy of Fallot, pulmonary atresia, and multifocal pulmonary blood supply seen at the Hospital for Sick Children, Great Ormond Street, and National Heart Hospitals since 1965, only 72 (33\%) arrived undiagnosed. The remainder were referred for specialist management by other cardiologists. Most of the 72 primary referrals required early surgery for critical cyanosis $(65 \%)$ or heart failure $(18 \%)$, but $12 \%$ had staging operations aimed at eventual "complete repair". Actuarial survival was $63 \%$ at 1 year, $52 \%$ at 5 years and $25 \%$ at 20 years. The early presentation and huge early surgical risk means that the applicability of the ambitious surgical manoeuvres often reported may be limited when one considers the whole spectrum of the patients.

Staged strategies aimed at eventual "complete repair" concentrate on manoeuvres to encourage growth of the central pulmonary arteries; and unifocalisational procedures to minimize sources of pulmonary flow and reduce the risk of pulmonary vascular obstructive disease. If a satisfactory pulmonary circulation can be established, "complete repair" involves insertion of a conduit between the right ventricle and the pulmonary arteries and closure of the ventricular septal defect. Late results of the newer protocols which claim to offer the prospect of this definitive repair to a high proportion of patients are not yet available. A high postoperative right ventricular to left ventricular pressure ratio is known to increase both early and late mortality and the late functional result is worse, as after repair of tetralogy of Fallot. This is due to increased afterload on the right ventricle on the setting of a closed ventricular septal defect. In addition, the insertion of an extracardiac conduit makes further surgery inevitable, as all conduits in children have been shown to require replacement for degeneration or growth mostly within 10 years. In older patients, results of homograft insertion has been much more satisfactory, with Ross and Somerville reporting $88 \%$ actuarial survival up to 18 years.

Long-term follow-up of the patients now undergoing complex stage surgical protocols for tetralogy of Fallot with pulmonary atresia will be fascinating over the next few years. It will be instructive to compare the results of these strategies aimed at definitive repair to simpler palliation protocols with eventual goal of heart and lung transplantation.

\section{Arrhythmias in postoperative/intensive care} John E. Deanfield

\section{Hospital for Sick Children, London}

Cardiac arrhythmias in early postoperative patients may result from: temporary or permanent damage to the conduction tissue and myocardium at surgery; electrolyte and ventilation disturbances; use of inotropes; or preoperative predisposition to bradycardias or tachycardias due to the underlying cardiac abnormality such as isomerism of the atrial appendages, pre-excitation in congenitally corrected transposition or Ebstein's malformation. With improved understanding of the disposition of the conduction system, complete heart block is now rare. If it persists for longer than two weeks, permanent pacing is required. Occasional pacing is indicated for sinus or junctional bradycardias after operations such as intra-atrial repair of complete transposition or, rarely, closure of atrial septal defect.

Tachyarrhythmias are commoner and the etiology is often multifactorial. The approach to management is the same as in other situations: accurate documentation and diagnosis, correction of predisposing factors and specific therapy if required. A surface electrocardiogram with multiple leads is necessary and it is extremely helpful if both temporary atrial and ventricular electrodes have been implanted by the surgeon in any case at risk. An atrial electrogram from an atrial electrode or transesophageal lead may clarify the relationship between atrial and ventricular activity. Further demonstration of the underlying atrial activity and relationship between atrial and ventricular activation may be facilitated by administration of the very short-acting atrioventricular nodal blocking agent adenosine, which at the same time may cardiovert the arrhythmia. Once the diagnosis is established, the choice of appropriate pharmacological treatment, cardioversion or overdrive pacing will depend on the degree of hemodynamic embarrassment secondary to the arrhythmia.

His bundle tachycardia or junctional ectopic tachycardia is a common potentially serious arrhythmia complicating congenital heart surgery, particularly after operations in the region of the atrioventricular node (repair of ventricular septal defect, atrioventricular septal defect, tetralogy of Fallot, and so on), but also occasionally in other situations, such as after the Fontan procedure. It is characterized by narrow complex tachycardia with atrioventricular dissociation and associated with beat-to-beat variation and blood pressure. This picture may be complicated by presence of bundle branch block. It is often missed and may cause profound hemodynamic deterioration and death. Treatment may involve: cooling of the patient; drug therapy with amiodarone or propafenone (likely to slow the tachycardia rather than convert it); and overdrive or $\mathrm{R}$ wave synchronized pacing. If hemodynamic stability can be achieved, sinus rhythm returns in the vast majority of patients spontaneously within a few days after surgery. Very occasionally $\mathrm{His}$ bundle ablation and permanent pacing has been performed for resistant cases. Other forms of ventricular tachycardia are rare.

\section{Use of inotropes in pediatric intensive and postoperative cardiac care G. William Henry \\ University of North Carolina, Chapel Hill}

Contractility and its effect on ventricular performance can be considered independently from preload and afterload. Defined most simply, contractility is the ability of the cardiac muscle to develop tension. Factors affecting contractility include the autonomic nervous system, metabolic/acid-base milieu, contractile mass, intrinsic properties of the contractile unit (including maturational development), endogenous/exogenous catecholamines and other pharmacologic agents. Inotropic agents are used to improve cardiac efficiency and to increase cardiac output by augmenting myocardial contractile force by increasing the availability of ionized cytoplasmic calcium for interaction with the contractile proteins (actin and myosin) in the myocyte. This interaction within the sarcomere is a complex process involving the regulatory proteins tropomyosin, troponin $\mathrm{T}$, troponin I, and troponin $\mathrm{C}$.

Classes of inotropes include cardiac glycosides, adrenergicagonists, 
phosphodiesterase inhibitors, adenyl cyclase agonists, and bipyridinenonglycoside-sympathomimetic compounds. The most commonly used inotropes in clinical practice include cardiac glycosides (such as Digoxin) and catecholamines/sympathomimetic amines (such as epinephrine, dopamine and dobutamine). Newer agents from the bipyridine-nonglycoside-sympathomimetic compounds (for example, amrinone) are establishing a role in pediatric cardiac intensive care as effective inotropes and afterload reducing agents. Agents that modulate the sensitivity of the contractile proteins of the myocyte to ionized calcium, along with agents that modulate fast sodium channels, offer a potential source of newer classes of inotropes for clinical use. The development of agents that augment diastolic function offers another potential method for improving myocardial contractility.

Noninvasive diagnosis of atrioventricular septal defects with concordant atrioventricular and ventriculoarterial connections G. William Henry

University of North Carolina, Chapel Hill

The diagnosis of atrioventricular septal defects necessitates recognition of the anatomic features arising from the deficiency of the atrioventricular septum coupled with a description of the physiologic consequences of these anatomic features. Cross-sectional and color-flow imaging offer a noninvasive method for obtaining such information.

Deficiency of the atrioventricular septum results in a discrepancy between the ventricular inlet and outlet dimensions which can be demonstrated easily in the parasternal long-axis view. The deficiency of the septum also results in a common atrioventricular junction that is seen in four-chamber views, especially from an apical cut. A further consequence of this common atrioventricular junction is the anterior displacement ("unwedging") of the aorta that contributes to the long subaortic outflow tract (demonstrated easily in a parasternal short-axis cut). The atrioventricular valvar anatomy is also typical, consisting of five leaflets, two bridging the ventricular septum, two confined to the right ventricle and one to the left ventricle. The resulting trifoliate arrangement of this valvar anatomy in the left ventricle distinguishes from the normal mitral valve.

Characterization of the atrioventricular orifices (common versus separate) and the relationship of the bridging leaflets to the septal structures ("floating" versus attachment to ventricular or atrial septums) define in large measure the expected physiologic consequences for interatrial interventricular, and/or ventriculoatrial shunting - which can be demonstrated by cross-sectional imaging and color-flow mapping. Further characterization of the elongated left ventricular outflow tract by Doppler interrogation and characterization of the ventricular balance or dominance by crosssectional imaging is important in defining physiologic determinants of the natural history.

\section{Complete transposition with ventricular septal defect- complex repair \\ Roger B. B. Mee \\ Royal Children's Hospital, Melbourne}

The ideal time for anatomical repair for transposition with ventricular septal defect is open to debate. At the Royal Children's Hospital, the protocol for timing is based on the degree of congestive heart failure. If the patient is intensive care dependent, then anatomical repair is performed within a day or two. If the patient is independent of hospital then anatomical repair is performed at about 2 months of age. If the patient is dependent on ward care, (nasogastric feeding), then the timing of repair lies somewhere in between those intensive care dependent and those independent of hospital. The presence of coarctation of the aorta with arch hypoplasia or interruption, requiring prostaglandin infusion, has been an indication for semiurgent repair of all defects through midline sternotomy.

The surgical technique for neonates includes single venous cannulation, performing the arterial switch under full cardiopulmonary bypass at $22^{\circ} \mathrm{C}$. The septal defects are then closed with a period of circulatory arrest at $18^{\circ} \mathrm{C}$ nasopharyngeal. If interruption of the aorta exists, or coarctation with arch hypoplasia, then the aortic arch is repaired, first under complete circulatory arrest or using our technique of isolated myocardial perfusion. The ventricular septal defect is always closed using the transatrial approach. If there is a large discrepancy in size between the ascending aorta and the pulmonary trunk, then the pulmonary trunk is transected a few millimeters higher than usual and gathered in to the smaller distal ascending aorta without addition of a gusset. By higher transection we hope to avoid bringing in the pulmonary valvar commissures and making the pulmonary valve incompetent. If the aortic arch has had to be repaired at the same time, then latterly we are inclined to excise the small distal ascending aorta and anastomose the proximal pulmonary trunk to the combined high ascending aorta and descending aorta (which has been anastomosed to the underside of the arch). This avoids leaving a hypoplastic segment of ascending aorta in the repair. When interruption or coarctation is present the potential for outflow tract obstruction in the right ventricle is assumed and a resection of subaortic muscle bar is usually performed through the aortic valve and or through the tricuspid valve at the time of repair.

The timing of intervention for the Taussig-Bing malformation is similar to that for transposition with ventricular septal defect. In our experience, there has been quite a high incidence of associated arch obstruction and obstruction of the right ventricular outflow tract requiring simultaneous attention. The arterial switch, when great vessels are side-by-side, is a little more difficult but the LeCompte manoeuvre is performed as with all arterial switches. We are inclined towards closure of the leftward portion of the pulmonary trunk and anastomosis of proximal aorta to rightward pulmonary trunk and right pulmonary artery directly.

Complete transposition-surgical techniques with coronary arteries

Roger B. B. Mee

Royal Children's Hospital, Melbourne

The basic technique used at the Royal Children's Hospital is the creation of two medially based trapdoor flaps in the proximal pulmonary trunk. The flaps in effect wrap around the D-shaped coronary cuff and are sewn back along the top edge for abour a third of the distance creating two dog ears. This roughly restores the circumference of the proximal pulmonary trunk bearing the two coronary arteries to what it was before developing the flaps. In addition, the wrap around flap creates a cone-like outpouching of the proximal neo-aorta with the coronary arising from the apex of the cone. The aorta and pulmonary trunk are transected about $2 \mathrm{~mm}$ above the commissures. When the circumflex branch arises from the right side, either on its own or as a branch of the morphological right coronary artery, then the pulmonary trunk is cut obliquely higher on the right side than the left. A medially based trapdoor flap created on the right side is therefore higher than on the left. Single coronary arteries have not been considered a problem and appear to have transposed well. Our practice has been to repair the aortic sinus defect with autologous pericardium before removing the cross clamp because access to the aortic sinus defect is a little more difficult once tone returns to the heart. For the single coronary arising from the right posterior sinus, with a left branch passing between the aorta and pulmonary trunk, the method for translocation has been described by Yacoub. The coronary button cannot be rotated, and 
autologous pericardium, or by using a flap of aortic sinus wall from the non-coronary sinus as suggested by Imai. If both coronary orifices arise from the right posterior sinus, but are separated by a millımeter, then they can be separated and treated in the usual fashion. This does involve taking down the commissure of the aortic valve between right and left sinuses and resuspending it to the pericardial patch repair of that sinus. An intramural left coronary artery behind the commissure between right and left posterior aortic sinuses can also be handled in the same way. Our experience of the intramural coronary artery is that the orifice is usually smaller than would be expected or else frankly stenotic. We have made a practice of unroofing the intramural coronary on the internal side of the aorta to relieve the stenosis and to create a larger cuff around the new orifice.

With transfer of the coronary arteries and end-to-end anastomosis between the proximal pulmonary trunk and the distal ascending aorta, requiring usually no more than 45 minutes of cross-clamp time, we have confidence that apparent myocardial failure on coming off cardiopulmonary bypass must be attributable to inadequate coronary blood flow from the translocated coronary arteries rather than primary myocardial insufficiency. Such a policy demands immediate revision of the kinked coronary artery and this can usually be achieved by closely studying the coronary arteries. Minor kinking can be handled by using a horizontal plicating mattress suture placed from the edge of the cuff to the neo-aortic adventitia in the direction opposite to the direction of kinking. Occasionally, a coronary artery has to be completely reimplanted. By using the trapdoor flap, rather than excising any pulmonary arterial wall, when relocation of the coronary is required, the flap is simply sewn back into place and the coronary button placed in a different position.

Although several techniques are described for coronary arterial translocation, it is our opinion that the trapdoor is suitable for the greatest proportion of coronaries. To date we have had no late deaths in arterial switch operation for simple transposition, no late deaths for transposition with ventricular septal defect, 1 late death for Taussig-Bing managed by arterial switch (recurrent mediastinal infection) and 2 late deaths after conversion of a Mustard or Senning operation to an arterial switch (one due to arrhythmia and one due to neo-aortic incompetence and myocardial failure in the presence of patent coronaries). In more than 90 postoperative catheter studies, we have identified no occluded coronary arteries.

\section{The Fontan procedure and the cavopulmonary connection Roger B. B. Mee}

Royal Children's Hospital, Melbourne

Incomplete cavopulmonary connection is in the form of unilateral or bilateral bidirectional superior cavopulmonary shunts. Complete cavopulmonary connection involves a bidirectional cavopulmonary shunt combined with a baffle to connect the inferior caval vein to the stump of the superior caval vein, which is then also anastomosed to the adjacent pulmonary artery. Complete cavopulmonary connection is therefore achievable in one or two stages. Partial cavopulmonary connection is reserved for those patients who, at the time of needing further intervention, are for various reasons considered unsuitable for a complete cavopulmonary connection. Usually this is because of young age, atrioventricular valvar regurgitation, obstructed pulmonary venous return, major pulmonary arterial distortions, or stenoses, or outflow obstruction to the ventricular mass. The partial cavopulmonary connection is therefore usually associated with some other procedure, or procedures designed to enhance the probability of the patient fulfilling Fontan criteria at a later date. Complete cavopulmonary connection is a modification of the Fontan principle, and is particularly advantageous when there is a single superior caval vein and in hearts where there is little space between the ascending aorta and the superior caval vein for a direct atriopulmonary connection. In addition, total cavopulmonary connection has the theoretical advantage of reducing the radius of curvature of atrial wall included in the connection between the inferior and superior caval veins, thus reducing the wall tension and the possibility of atrial arrhythmias. On the other hand, the total cavopulmonary connection involves an extensive atrial suture line reminiscent of that used in the Senning or Mustard operation and hence the reduction in atrial arrhythmias using this method may not be as dramatic as was hoped for. A theoretical advantage is of creating something closer to laminar flow and thus less energy wasted in conveying systemic venous blood to pulmonary arteries. In order to avoid a suture line that almost encircles each caval orifice we have made a slight modification to the baffle in that the anterior edge of the baffle is sewn into the atrial suture line which runs parallel and about a centimeter in front of the terminal crest. Other modifications of the connection have been described and of interest is that involving an atrial flap in place of a synthetic baffle. Neither of these latter two modifications are accompanied by any evidence as yet of a superiority over the original description of the operation.

When two superior caval velns are present, there is some concern that the smaller size of the caval vein on the side of the inferior caval vein may not afford a completely unobstructed connection to the pulmonary arteries. It is possible to enlarge a small superior caval veno-atrial junction by incising laterally behind the sinus node and creating an anastomosis with the pulmonary artery more distally. Such an incision is likely to interrupt the artery to the sinus node if it runs behind the cavoatrial junction. When two superior caval veins are present and there is interruption of the inferior caval vein with azygos or hemiazygos continuation to one of the superior caval veins, then the orifice of the superior caval vein on the side of the hepatic veins will probably be adequate for this reduced flow. Alternatively the hepatic flow can be left to mix with the pulmonary venous blood as described in the Kawashima operation.

For any patient series, utilizing the bidirectional cavopulmonary shunt as an intermediate operation before Fontan, we can probably make two assumptions: first, that some cases undergoing bidirectional cavopulmonary shunt could well have had a complete Fontan operation, and, second, some cases which would have been considered unsuitable for a Fontan, may subsequently be viewed as suitable. Insufficient time has gone by with use of the bidirectional cavopulmonary shunt to know just what percentage of otherwise unsuitable patients have been retrieved and turned into reasonable Fontan candidates.

\section{Control of infection in the intensive care unit \\ Roger B. B. Mee}

Royal Children's Hospital, Melbourne

Control of infection in the intensive care unit commences in the operating room with careful clean insertion of lines and careful attention to sterile technique in the operating room.

Prophylactic antibiotics probably play an important part in cardiovascular surgery, but their use should not be extended for too long postoperatively. In the intensive care unit, the principal source of infection is through penetrating lines and catheters. Intravenous lines with multiple ports are the main offenders, where as left atrial, pulmonary artery and arterial lines which have a slow incidence of port opening, are minimally incriminated. "No touch technique" handling of ports is probably important, along with the method of cleaning the ports with alcohol after each use. Hand washing is probably the single most important intervention to reduce infected lines and this had proved very difficult to implement. Hand washing 
must be conveniently done or it will be neglected and the accent on hand washing should be between handling of the skin or bodily orifices and handling of intravenous ports. The evils of overcrowding and understaffing are very well understood and well documented in intensive care units. Under conditions of both overcrowding and understaffing, hand washing suffers, "no touch techniques" suffer and cross contamination increases. Finally, much has been written about the use of antibiotics in the intensive care situation. It is clear that antibiotics should be used only for proven infections or in the patient who clinically appears to have a progressive infective process, but only after full culturing with a willingness to stop the antibiotics if the cultures prove negative over the next day or two. Antibiotics should not be used to prevent infections in the intensive care unit, only to treat them.

\section{Atrioventricular septal defects-repair of the left atrioventricular} valve

Roger B. B. Mee

Royal Children's Hospital, Melbourne

Since 1980, the policy at Royal Children's Hospital has been two patch repair of complete defects early in infancy. The common leaflets have not been divided. Considerable care has been taken to mark the exact points of apposition of common leaflets or the exact point of apposition of superior and inferior left septal components of the leaflets. The ventricular component of the defect is repaired first with a segment shaped patch of knitted Dacron sewn in along the septal crest with a running prolene suture. The suture is woven in and out between the cords on the right side of the septal crest in order to preserve the function of the right component of the common valve. Horizontal prolene mattress sutures are then passed through the free edge of the parch, through the bridging leaflets, or through the septal edge of the left septal components of the common valve before being passed through an autologous pericardial patch reinforced with a thin strip of Gore-tex. It is at this point that the pericardial patch is turned forward and the left component of the valve inspected. If there was regurgitation preoperatively it is usually through the "cleft" or septal commissure and in such cases the edges are usually thickened. The cleft or commissure is closed with interrupted surures, either completely if there is a well developed mural leaflet, or incompletely if the left mural leaflet has a short base. The degree of closure of the septal commissure depends on careful sizing of the valve according to a nomogram. We will accept a valvar orifice 1 millimeter smaller than the required size if it is to improve competence. The rule of closure may be broken when there is no preoperative regurgitation and the edges of the adjacent leaflets are very thin. Our feeling here is that sutures in non-thickened edges may not hold. Our first 62 infants undergoing two patch repair of complete defects were reviewed. Roughly the first half had the commissure left open and 7 of these needed reoperation and commissural closure. The second half had the commissure routinely closed and only one of these required reoperation for commissural closure. Of interest in this series was the fact that we could find no relationship between the degree of preoperative and postoperative valvar regurgitation. The overall reoperation rate after repair of complete atrioventricular septal defect in infants has been about $10 \%$ and the need for reoperation for recurrent left atrioventricular valvar regurgitarion is something we have not been able to eliminate. We suspect, therefore, that in at least $10 \%$ of our patients, the repair has not been ideal.

Surgical treatment of arrhythmias in childhood

G. K. Danielson

Mayo Clinic, Rochester

Operation for supraventricular tachycardia is mostly commonly performed for arrhythmias associated with one or more accessory atrioventricular conduction pathways. New methods of multipoint mapping with computerization are now available. Simultaneous bipolar electrograms can be digitized, analyzed, and then displayed in the operating room. A "sock" of bipolar electrodes is placed around the ventricles and recordings are made during sinus rhythm, atrial pacing, and induced tachycardia. A band can then be positioned in the atrial side of the atrioventricular groove to record atrial depolarization during ventricular pacing and tachycardia. Analysis of simultaneous recordings from multiple points is advantageous because only a few beats of tachycardia are needed for mapping, and manipulation of the heart is minimized. It is also easier to determine if the pathway is localized, a broad band, or multiple.

The pathways may be divided by an endocardial approach which utilizes cardiopulmonary bypass or an epicardial approach which may or may not utilize cardiopulmonary bypass. We have used the epicardial approach for some right-sided pathways, but find the endocardial approach more satisfactory for anterior and posterior septal pathways and left-sided pathways. From April 1972 to October $1,1987,309$ patients underwent evaluation for tachycardia due to accessory conduction pathways. One-third (103) were operated on. 52 of the 103 patients were age 6 months to 21 years. The mean duration of symptoms was 5.6 years and the mean number of medication trials was 2.4. One to three accessory pathways were documented, and additional cardiac defects including Ebstein's malformation, ventricular septal defect, and partial atrioventricular septal defect were repaired in 15 patients. There was no operative mortality and no complete heart block. In 51 patients, tachycardias were not inducible prior to hospital discharge. On late follow-up, only one patient had return of symptomatic preexcitation $(98 \%$ success rate).

Although medical treatment is highly successful in most cases of atrioventricular nodal reentry tachycardia, severe symptoms in some patients may require interventional treatment. Operations that have been developed to prevent reentry tachycardia without producing complete heart block include application of discrete cryolesions around the atrioventricular node and surgical dissection of the perinodal atrium. Our surgical experience includes 17 patients operated upon from January 1986 to March 1992. All patients survived without heart block. There was one late recurrence of tachycardia requiring antiarrhythmic drugs ( $94 \%$ success rate).

Supraventricular tachycardia due to ectopoic atrial focus is rare, but surgical treatment is rewarding because morbidity and mortality of operation are low and the chance of permanent cure is high. Patients with incessant automatic tachycardia develop congestive cardiomyopathy; heart failure is cured when the tachycardia is ablated. For patients with free wall or septal foci, wide excision with direct or pericardial patch closure of the atrium is satisfactory. Cryothermic ablation is a useful adjunct to excision. Our surgical experience consists of 13 patients from 1984 to March 1992. All patients survived operation and 12 are free of their ectopic arrhythmia (92\% success rate). The one exception had a right atrial cardiomyopathy.

Operation for ventricular arrhythmia is uncommonly required in the young. Surgery has been effective in treating ventricular tachycardias caused by cardiac tumors and those related to ventriculotomy scars following repair of congenital heart disease.

Surgical treatment of supraventricular and ventricular tachycardia in the young patient has been successful in our experience, with a mortality of 0 and success rates of 92 to $98 \%$, and the late results have been gratifying. Recently, atrial and ventricular arrhythmia have been approached with radiofrequency catheter ablation techniques, and early successes have been achieved. Further experience is necessary to determine the relative roles of these two important modalities for 
controlling the many forms of supraventricular and ventricular tachycardia.

\section{Ventricular assist devices \\ Roger B. B. Mee \\ Royal Children's Hospital, Melbourne}

The paediatric patient who fails to wean from cardiopulmonary bypass has only recently been offered a chance of meaningful prolonged cardiac or cardiorespiratory support, as has been available for adults for many years. The constrained vortex pump is probably the most versatile device allowing up to full cardiac output support for the full range of paediatric sizes. Most commonly, cardiopulmonary support has been used irrespective of satisfactory lung function. Extracorporeal membrane oxygenation is more complex than simple ventricular assist and carries a higher complication rate. Ventricular assist is probably all that is necessary in about two-thirds of patients requiring support and may be effective even in functionally univentricular hearts with venous mixing. At Royal Children's Hospital, 20 patients have received ventricular assistance, with 10 discharged from hospital. Eight received extracorporeal oxygenation subsequent to cardiac surgery with 1 survivor. By July 1991 in USA, 385 patients were supported by extracorporeal oxygenation after cardiac surgery with $33 \%$ discharged from hospital.

\section{The modified Fontan procedure for complex congenital heart} disease

G. K. Danielson

Mayo Clinic, Rochester

Our experience with the Fontan procedure for tricuspid atresia began in October, 1973. One year later, the concept of right heart bypass was extended to a patient who had double inlet left ventricle. Subsequently, the original Fontan operation was modified in several other important respects and has been extended to over 843 patients with all types of functionally univentricular heart.

Between 1973 and January 1, 1992, 202 patients with tricuspid atresia had a modified Fontan procedure. Age range was 7 months to 42 years with 46 patients (23\%) sixteen years old or older; 196 patients had received from 1 to 4 prior cardiovascular surgical procedures. Hospital mortality rates were $17 \%$ ( 9 of 54 ) from 1973 $1980,9 \%$ (9 of 104) from 1981-1987, and $2 \%$ ( 1 of 44) from 1988 1992. There have been 13 late deaths. Follow-up of 166 survivors (3 months to 18 years, median 6.9 years) showed $90 \%$ to be in excellent or good condition and $10 \%$ to be fair or poor condition. The two major factors that influenced operative and late results were pulmonary arteriolar resistance and left ventricular function. A catheterization index devised by adding pulmonary arteriolar resistance to left ventricular end-diastolic pressure divided by pulmonary plus systemic blood flow is helpful in selecting candidates mostly likely to survive and benefit from the Fontan operation. If this index is less than 4.0 , then the postoperative right atrial mean pressure will be $20 \mathrm{~mm}$ of $\mathrm{Hg}$ or less, a circumstance associated with $95 \%$ early and $90 \%$ late survival rates.

Between 1974 and January 1, 1992, 187 patients with double inlet left ventricle had a modified Fontan procedure. Age at operation ranged from 23 months to 41 years (median, 10 years); 135 patients had received from 1 to 3 prior cardiovascular surgical procedures. Concomitant procedures included subaortic resection $(n=22)$, pulmonary arterial reconstruction $(n=18)$, atrioventricular valvar repair $(n=6)$ and aortic valvar replacement $(n=1)$. Hospital mortality rates were $21 \%$ ( 8 of 39 ) from $1974-1980,11 \%$ ( 10 of 93) from 1981-1987, and 5\% (3 of 55) from 1988-1992. The 18 late deaths were due to reoperation $(n=8)$, myocardial failure $(n=6)$, sudden arrhythmia $(n=3)$, and bleeding varices $(n=1)$. Neither hospital nor late mortality rate was significantly related to age at operation. Follow-up of 140 survivors ( 4 months to 13 years, median $=5.6$ years) showed $89 \%$ to be in excellent or good condition and $11 \%$ to be in fair or poor condition.

Surgical results for more complex forms of "univentricular" heart are also improving with increasing experience. For example, mortality in asplenia and polysplenia syndromes has been reduced from $56 \%$ in the early experience to $8 \%$ in the recent experience in our series of more than 75 patients. Survival curves have now been constructed. Survival at 12 years is $8.4 \%$ better for tricuspid atresia than double inlet left ventricle and $15.3 \%$ better for tricuspid atresia than more complex forms of "univentricular" heart. If patients with high pulmonary arteriolar resistance and poor ventricular function are excluded, 12-year survival rates of $90 \%$ or better can be expected. In spite of potential problems, including pulmonary arteriovenous fistulas, protein-losing enteropathy, and atrial arrhythmias, the modified Fontan procedure represents a significant advance in the treatment of congenital heart disease. This procedure provides effective palliation for functionally univentricular anomalies which have no other satisfactory treatment. Criteria for operability have been extended beyond those originally proposed for this operation. The operative mortality continues to decline with increasing experience, late results are good, and longevity is increased.

\section{Pulmonary atresia intact ventricular septum-protocols for treatment \\ Roger B. B. Mee \\ Royal Children's Hospital, Melbourne}

For the last 12 years, our treatment protocols have been based largely on the presence or absence of a well-formed infundibulum. Latterly this has been exclusively diagnosed by ultrasound. The well-formed infundibulum is associated with membranous pulmonary valvar atresia and a well formed pulmonary trunk down to the atresia. With a hypoplastic, or absent infundibulum, the pulmonary trunk is tapered, and there is usually an element of muscular atresia in the infundibulum as well. In this latter group, there is a much higher incidence of fistulous connections with the coronary arteries. These have been defined as major if there is retrograde filling of the aortic root on right ventricular injection at subsequent catheter studies, or minor if there is some filling of coronary arteries without opacification of the aortic root. In patients with a well formed infundibulum, only three cases of minor fistulous connections have been identified and no major connections. We believe that even in those patients with absent infundibulum and very small ventricular cavities, complete absence of the trabecular portion is extremely rare, emphasizing that those using the tripartite definıtion should appreciate that the mural hypertrophy squeezes out the cavity. It also appears that on the whole the patients with poorly formed infundibulum have small tricuspid valves. Our treatment protocol is, therefore, directed towards achieving a biventricular repair in those patients with well formed infundibulum, and accepting that a Fontan type of repair is the fate of those patients with a poorly formed or absent infundibulum. At neonatal presentation, the need to exhibit prostaglandin $E_{1}$ infusion for a closing duct is noted, and it is assumed that the duct will largely close after prostaglandin is withdrawn. On the other hand, for those presenting with an open duct (some times at a later age), no such assumption that the duct will close is made. The interatrial septal defect is assessed, and if there is any doubt about its size, then a balloon atrial septostomy is performed.

Those patients presenting with a well formed infundibulum go forward to left thoracotomy where open valvotomy is performed without bypass through the pulmonary trunk and a PTFE shunt is placed from the subclavian artery on the left to the pulmonary trunk and the duct is ligated. In the group of patients presenting with a 
poorly formed or absent infundibulum, a right PTFE shunt is performed if the patient presented with a closing duct. If the duct was wide open on presentation (without prostaglandin $\mathrm{E}_{1}$ infusion), then a PTFE shunt is placed from the left subclavian artery to the proximal left pulmonary artery, or the pulmonary trunk if it is of reasonable size, and the duct is ligated. Since 1980 through to 1992, 48 neonates have presented with pulmonary atresia, intact septum. Thirty-one have a well-formed infundibulum and there has been 1 perioperative death from an initial palliation. Seventeen patients had absent or poorly formed infundibulum and there were 2 perioperative deaths from initial pallation.

At follow-up catheter investigation in patients with a well-formed infundibulum, if balloon occlusion of the interatrial communication causes no rise in right atrial pressure, and the tricuspid valve and the right ventricle appear to be of adequate size, then a biventricular repair is performed. So far 12 patients have undergone biventricular repair with 1 late death at 4 months, possibly from aspiration pneumonia. If the left ventricle is not considered adequate as a pump, then an intermediate procedure may be performed, which includes repeat valvotomy, infundıbular resection, tricuspid valvoplasty and resection of muscle from the trabecular pouch. The interatrial communication and the shunt are left for the time being. Five such procedures have been performed with 2 going on to biventricular repair. Only 1 patient with a well formed infundibulum has undergone a Fontan repair because of prolonged failure of adequate growth of the right ventricle and tricuspid valve. In those patients with absent or poorly formed infundibulum, our management tracking is towards a Fontan operation. If initial palliation is failıng, and the patient does nor fulfil the criteria for Fontan, then a bidrectional cavopulmonary shunt may be performed as an intermediate procedure. Ten of the 15 survivors of initial palliations in patients with absent or poorly formed infundibulum have gone on to a Fontan type operation with 2 early deaths. Both early deaths seem to have been associated with myocardial ischemia. At the time of the Fontan operation, we leave the right ventricle and tricuspid valve intact and design the Fontan repair so that the right ventricle fills with pink blood.

Surgical repair of Ebstein's malformation

G. K. Danielson

Mayo Clinic, Rochester

From April 1972 to October 19, 1992, 232 patients with Ebstein's malformation underwent repair. Results in the first 189 patients have been reviewed. Ages ranged from 11 months to 64 years (median 16 years, mean 19.1 years). In $58.2 \%$, tricuspid valvar reconstruction was possible and in $36.5 \%$, a prosthetic valve, usually a bioprosthesis, was inserted. In $5.3 \%$, a modified Fontan or other procedure was performed. There were 12 hospital deaths (6.3\%). All 28 patients who had accessory conduction pathways (WolffParkinson-White syndrome) underwent successful ablatıon of the pathways as part of the operative treatment. Follow-up was obtained in $151(85.3 \%)$ patients. Of these patients more than one year postoperative, $92.9 \%$ were in New York Heart Association class I or II. There were 10 late deaths, seven cardiac (four sudden), two noncardiac, and one of an unknown cause. Postoperative echo-Doppler assessment showed the atrial septum was intact in all patients and tricuspid valvar function was good to excellent in most parients. Four of the 110 patients (3.6\%) who underwent valvar reconstruction required reoperation 1.4 to 14.1 years later. Postoperative reduction in heart size was usual, atrial arrhythmias were reduced, and late postoperative exercise testing showed a significant improvement in performance; maximal oxygen consumption increased for a mean of $47 \%$ of predicred value before operation to a mean of $72 \%$ after operation. Nine patients had a total of 12 successful pregnancies with deliveries of normal children.

Tetralogy of Fallot with pulmonary atresia-steps in palliation Roger B. B. Mee

Royal Children's Hospital, Melbourne

We define two groups of patients, those with a duct-dependent circulation who undergo a right PTFE shunt as initial palliation, and those of a much more complex nature with small pulmonary arteries and supply to the lungs via collateral arteries. This second group is subdivided into two further groups. Those with complete absence of any central pulmonary arterial system are handled by creating pericardial rolls in each hemi-thorax. The supply from collaterals is detached from the aorta and anastomosed to the pericardial roll which is also supplied with a PTFE shunt from the ipsilateral subclavian artery. These rolls are made of sufficient length, such that they can be mobilized from the front and joined together behind the superior caval vein and the ascending aorta at the time of complete repair. In the second subgroup, where confluent central pulmonary arteries are identified, however small, our approach is to stimulate growth of these arteries by shunting in to them. In very small pulmonary arteries, we believe that peripheral shunts may be counter productive in terms of creating a high incidence of pulmonary arterial branch distortion. Our preference would be a central shunt for these patients. For the very small pulmonary arteries, we would favour as end-to-side shunt from the pulmonary trunk to the ascending aorta and, for the somewhat larger ones, a central shunt using PTFE tube graft. Our preference is to embark on shunting within the first year of life. Having demonstrated some growth in size of the pulmonary arteries we then proceed with unifocalisation through each hemi-thorax avoiding the use of prosthetic material whenever possible. On the right side, a reversed azygos vein can be used as a conduit, particularly where there is a right-sided descending aorta and the length of the collateral arteries is too short to be directly anastomosed to the central right pulmonary artery. Even growth of very small pulmonary arteries from central shunting occurs in only about $50 \%$ patients. Following the first 6 patients entering our program, who had an outflow patch placed across the outflow from the right ventricle to the pulmonary trunk, we have abandoned this as an initial procedure to stimulate pulmonary arterial growth. At the time of completing the dynamic repair, repair of residual more centrally placed branch pulmonary arterial stenosis is relatively easily achieved from a midline sternotomy. The fate of the central end-to-side shunt from the pulmonary trunk to the ascending aorta in very small pulmonary arteries is reviewed in this presentation.

\section{Tetralogy of Fallot-surgical techniques}

Roger B. B. Mee

Royal Children's Hospital, Melbourne

Since early 1981, at Royal Children's Hospital, all repairs of tetralogy of Fallot have been achieved using the transatrial transpulmonary approach. Infundibular resection is performed through the tricuspid valve to the level of the pulmonary valve and the ventricular septal defect is repaired through the tricuspid valve using a knotted Dacron patch secured with interrupted Teflon pledgeted, horizontal Prolene mattress sutures. The pulmonary valve is then sized through the tricuspid valve and, if adequate, then no approach through the pulmonary trunk is required. Ifinadequate, then a vertical incision is made in the pulmonary artery, a full valvotomy performed without peripheral release, and the "annulus" remeasured. If it is still too small, then the "annulus" is incised in line with an anterior commissure, preserving the leaflets and extending the incision for a few millimeters into the outflow tract. Further infundibular resection may be performed from above. The pulmonary trunk, with or without the distal infundibulum, is then repaired 
with an autologous non-treated pericardial patch if the branch pulmonary arteries are well formed. If pulmonary arteries are on the small side, then the pericardial patch is supplemented by an autologous non-treated pericardial monocusp. It is recognized that this monocusp does not last for very long, in that it tends to adhere to the outflow patch. We believe it is useful, nonetheless, in promoting a smooth postoperative course. Proximal branch pulmonary arterial stenosis is also repaired with autologous pericardium. The whole repair is almost always performed on full cardiopulmonary by pass using the right angle metal cannulas inserted directly in the caval veins. When a left superior caval vein is present draining to the coronary sinus, this is also cannulated. Increasingly, we are debulking the coronary sinus which we feel may be partially obstructive to the mitral valve. This is achieved by removing the roof and resewing the edges together. Again the coronary sinus is approached through the right atrium by mobilizing the interatrial septum. With cannulation of the left superior caval vein, access to the pulmonary arteries for repair is difficult so, after closure of the right atrium, the cannula in the vein is removed and placed in the right atrial appendage and we can proceed to repair in the usual way with the heart beating during rewarming.

\section{The morphology of tetralogy of Fallot}

\section{Robert H. Anderson}

\section{National Heart \& Lung Institute, London}

The essence of tetralogy of Fallot is antero-cephalad deviation of the muscular septum separating the subpulmonary and subaortic outlets. This outlet septum, inconspicuous in the normal heart, becomes recognized as a structure in its own right in tetralogy, located exclusively within the right ventricle, and inserted above and in front of the anterior limb of the septomarginal trabeculation. Septal malalignment in itself, nonetheless, is insufficient to account for the anatomy. The outlet septum can be deviated and attached in similar fashion when the subpulmonary outlet is unobstructed-the socalled Eisenmenger complex. Thus, in addition to septal deviation, it is also necessary to find hypertrophy of the anteriorly located septoparietal trabeculations to make the diagnosis of tetralogy.

At one stroke, the septal malalignment accounts for the presence of subpulmonary obstruction, the attachment of the leaflets of the aortic valve within both ventricles ("aortic overriding"), and the presence of a cone of space subtended by the attachment of the aortic valvar leaflets to the crest of the ventricular septum. Any plane within this cone can justifiably be nominated as the ventricular septal defect, but the right and left ventricular margins are of most surgical importance. The right ventricular margin is the locus for placement of sutures during surgical repair. This border is usually made up in part by fibrous continuity between the leaflets of the aortic and tricuspid valves (perımembranous defect). The conduction axis penetrates through this area as it joins the central fibrous body. Less frequently, in about one-fifth of cases, the border have be exclusively muscular. This arrangement protects the axis responsible for atrioventricular conduction. The margin can also vary when the outlet septum is absent and there is fibrous continuity between the leaflets of the aortic and pulmonary valves. In absence of the outlet septum, however, it is arguable as to whether a case truly shows tetralogy of Fallot. The subpulmonary obstruction, produced primarily by the "squeeze" between deviated outlet septum and hypertrophied septomarginal trabeculations, can be exacerbated by obstruction within the apical part of the right ventricle, at the level of the pulmonary valve, or within the pulmonary arterial tree. The attachment of the overriding aortic valve can vary between the extremes of concordant and double outlet ventriculo-arterial connections. Right ventricular hypertrophy is an acquired consequence of the deranged hemodynamics. Many associated lesions can accompany this anatomy.
Particularly important ones are straddling tricuspid valve, atrioventricular septal defect, second muscular ventricular septa defect, rudimentary formation of the leaflets of the pulmonary valve ("absent" valve), coronary arterial anomalies, right aortic arch, and pulmonary atresia instead of stenosis.

\section{Tetralogy of Fallot with pulmonary atresia} Robert H. Anderson

National Heart \& Lung Institute, London

Pulmonary atresta can appear in many anatomic guises-most notably with an intact ventricular septum or with an interventricular communication. Further variability is to be found in the presence of a ventricular septal defect, but the most important is the source of pulmonary arterial flow. This can be derived from several sources, including the important systemic-to-pulmonary collateral arteries. The latter vessels are almost always, but not invariably, found when the intracardiac anatomy is that of tetralogy of Fallot but with atresia instead of stenosis of the subpulmonary outflow tract. The atresta can be produced by muscular atresia or an imperforate valvar membrane, while the pulmonary trunk and intrapericardial arteries can themselves rarely be totally lacking. The ventricular septal defect can show all the variation to be found in tetralogy with pulmonary stenosis, including absence of the outlet septum when the infundibular morphology is more akin to common arterial trunk than tetralogy.

The anatomic focus in this lesion is on the pulmonary arterial circulation. Although systemic-to-pulmonary collateral arteries are most important, a significant number of cases receive the arterial supply via unilateral or bilateral arterial ducts. Almost always in such cases the pulmonary arteries themselves supply the entirety of the pulmonary parenchyma. More rarely the supply can be via an aortopulmonary window, the fifth aortic arch, or fistulas between the coronary and pulmonary arteries. There can also be acquired collateral supply. The congenital collateral arteries, when present, are usually found in cases with gross abnormalities in the arborization of the intrapericardial pulmonary arteries. Almost always the socalled "central" pulmonary arteries co-exist with the collateral arteries and are then fed at one or more points by the collateral arteries. Only rarely do collateral arteries co-exist in the same lung with a patent arterial duct. The collateral arteries vary in number from two to six, and usually arise from the descending aorta, although they can take origin from the brachiocephalic arteries or even from the coronary arteries. Some collateral arteries run to the lung hilum and then continue to supply a given area of the parenchyma without forming anastomoses. More frequently, the collateral arteries anastomose with the central pulmonary arteries so as to supply the entire area of parenchyma fed by the intrapericardial arteries. These anastomoses can be at hilar, segmental or intrapulmonary levels. In other instances, segments of lung can receive supply both from non-communicating collateral arteries and from intrapericardial pulmonary arteries. The relationship between the collateral arteries and bronchial arteries has yet to be decided. Some of the collateral arteries branch in fashions remarkably reminiscent of the normal bronchial arteries, and careful dissections show that the collateral arteries supply nutritive branches to the bronchial tree in addition to feeding the pulmonary arteries.

The anatomy of Ebstein's malformation Robert H. Anderson Nattonal Heart \& Lung Institute, London

The essence of Ebstein's malformation is presence of the hingepoint of some part of the leaflets of the tricuspid valve from the wall of the right ventricle rather than originating at the atrioventricular junction. This pathognomonic feature is then compounded by marked 
variability in the distal attachments of the leaflets. The normal tricuspid valve has septal, antero-superior, and mural or inferior leaflets. The septal leaflet, as its name suggests, is tethered by numerous cords to the inlet component of the septal surface of the right ventricle. The antero-superior leaflet is tethered focally between the medial and anterior papillary muscles, forming a curtain between the inlet and outlet ventricular components. The mural leaflet is tethered along the diaphragmatic surface of the ventricle, extending from the crux to its commissure with the antero-superior leaflet. In Ebstein's malformation, it is almost always the hingepoints of the septal and mural leaflets which are downwardly displaced, with maximal displacement being at the commissure between them at the crux. In many cases the septal leaflet can be partially or completely absent. Only rarely will the hingepoint of the antero-superior leaflet be displaced from the atrioventricular junction down the ventriculoinfundibular fold. Although the characteristic pathology is found at the hingepoint, it is possibly the variability in distal attachment which colours the clinical presentation of these cases. In some instances, the antero-superior leaflet retains its focal attachments. More frequently, abnormal tongues are formed connecting the antero-superior leaflet with the other leaflets, particularly the septal one, and then the leading edge of the leaflets and tongues obtain either hyphenated or linear attachments to a prominent muscular shelf formed between inlet and apical trabecular components of the right ventricle. It is as if the valve is trying to form a competent mechanism at the inlet-trabecular junction rather than at the atrioventricular junction. In the most severe cases the valve can be imperforate, thus producing tricuspid atresia. In less severe, albeit severely abnormal, cases the inlet component of the right ventricle can become markedly thinned and dilated-so-called anatomic atrialization. The outlet component of the ventricle, which together with the apical component forms the functional ventricle, also shows abnormal anatomic changes and is often hypoplastic. Associated malformations are frequent, and include pulmonary stenosis or atresia, atrial or ventricular septal defects and, more rarely, atrioventricular septal defect. Associated pulmonary atresia is now known to be a particularly potent lesion presenting during fetal life with gross dilatation of both the inlet part of the right ventricle and the right atrium. Ebstein's malformation is a frequent finding in hearts with discordant atrioventricular connections where, with usual atrial arrangement, it afflicts the left-sided atrioventricular valve.

\section{The anatomy of complete transposition \\ Robert H. Anderson}

National Heart \& Lung Institute, London

The essence of complete transposition is the combination of discordant ventriculo-arterial with concordant atrioventricular connections. This can be found with usual or mirror-image atrial arrangements. The discordant ventriculo-arterial connections ("transposition") can be found with other atrioventricular connections (discordant, double inlet or absence of the connection) or with isomerism of the atrial appendages ("heterotaxy") but these combinations have quite different clinical features. The connections found in complete transposition (very rarely produced also by discordant atrioventricular and concordant ventriculo-arterial connections) produce systemic and pulmonary circuits in parallel as opposed to the usual arrangement which is in series. The abnormal connections themselves impose a particular anatomy which can then be further varied by the presence of associated malformations.

The overall anatomy dictates the disposition of the conduction tissues (basically normal) and the coronary arteries. The arteries arise always from one or other, or more usually both, of the aortic sinuses which face the pulmonary trunk. These sinuses are best named according to whether they are to either the right or left hand of the observer standing within the non-facing sinus of the pulmonary trunk and looking towards the aorta, since this convention is independent of the relationship of the arterial trunks themselves. The different patterns can then be accounted for by describing origin of the right, circumflex and anterior descending arteries from either sinus, and supplementing this be describing their course relative to the arterial pedicles and also noting the origin and course of the artery to the sinus node. The most common variant, found in two-thirds of cases, is for the right coronary artery to arise from the righthand sinus and the other arteries to originate from the lefthand sinus. The marked variability in relationship of the arterial trunks is accounted for by describing the origin of the aorta relative to the pulmonary trunk in right-left and antero-superior orientations. There is also variability in infundibular morphology, although usually there is a subaortic infundibulum.

The important associated malformations are a ventricular septal defect, which shows the same variation as found in the normally connected heart, obstruction of the left ventricular outflow tract and other lesions such as persistent patency of the arterial duct or obstruction of the right ventricular outflow tract and aortic coarctation. Any lesion, however, must be anticipated. One important type of ventricular septal defect is characterized by malalignment of the outlet septum into the right ventricle with overriding of the pulmonary valve. This combination, which shows a spectrum towards double outlet right ventricle with subpulmonary ventricular septal defect, can be considered to represent the Taussig-Bing malformation. Malalignment of the outlet septum into the left ventricle, with subpulmonary obstruction and overriding of the aortic valve, sets the scene for the Rastelli procedure.

\section{The anatomy of valvar disease in children}

Robert H. Anderson

Natıonal Heart \& Lung Institute, London

The pathology of valvar disease is best understood by assessing how disease afflicts the various anatomic components of the valves, which divide conveniently into artertal and atrioventricular patterns. Both are complex anatomic structures. The atrioventricular valves have a junctional attachment (the annulus), leaflets, and tension apparatus supporting the leaflets. The leaflets are the individual parts of the sleeve of fibrous tissue which opens and closes to form the valvar mechanism. They are separated one from the other by the commissures, which fit snugly together when the valve is closed. Strictly speaking, a valve with only two leaflets (the mitral valve) can have only one commissure! Be that as it may, disease can afflict the annulus, the leaflets or the tension apparatus to produce stenosis, incompetence or both. Commissural fusion is the most typical cause of stenosis, and can be congenital or acquired. Annular dilatation or abnormalities of leaflets and tension apparatus, notably prolapse, are the common cause of incompetence, and again can be congenital or acquired. The most common acquired lesion is rheumatic fever which usually afflicts the mitral rather than the tricupsid valve. Congenital lesions can afflict the different components of either valve, but sometimes, as with Ebstein's malformation, show a propensity for one of the valves, although a similar lesion can affect the mitral valve. The congenital lesions include complete absence of the connection or imperforate leaflets, both producing valvar atresia, miniaturization and stenosis, and other lesions such as the parachute and arcade lesions.

The arterial valves also have a complex anatomic arrangement involving interaction of the sinuses of the arterial trunks with the supporting ventricular structures. Unlike the atrioventricular valves, there is no circular structure ("annulus") supporting the entirety of 
the hingepoints of the leaflets of the valves. Instead, the leaflets are attached in semilunar fashion with this arrangement incorporating a small part of the ventricle within the arterial sinus while parts of the arterial wall become incorporated into the ventricle as the interleaflet triangles. The valvar leaflets meet together at the commissures, with the peripheral attachment of each commissure being at the sinutubular junction. As with the atrioventricular valves, stenosis is produced by commissural fusion, which can be congenital or acquired. Incompetence is more usually due to dilatation of the ventriculoarterial junction, but can be the consequence of congenital or acquired deformation of the valvar leaflets. The most common acquired disease is rheumatic, with the aortic valve being most frequently involved. Congenital disease in pulmonary position usually produces a domed "acommissural" membrane which can be imperforate. In aortic position, it is more frequent to find either the so-called unicommissural valve, or the bicuspid variant. The unicommissural variant is formed on a tricuspid template, with grossly abnormal attachment of the leaflets. The bicuspid variant usually does not become diseased until adult life.

\section{The anatomy of atrioventricular septal defect}

Robert H. Anderson

National Heart \& Lung Institute, London

The normal heart has well-formed atrioventricular septal structures separating the right atrium from the subaortic left ventricular outflow tract. These are muscular, as a consequence of offsetting of the hingepoints of the mitral and tricuspid valves, and membranous, that part of the membranous septum above the level of attachment of the septal leaflet of the tricuspid valve. These septal areas can be absent entirely or in part in lesions such as double inlet ventricle or absence of one atrioventricular connection. The membranous atrioventricular septum can itself also very rarely be deficient in isolation. More importantly, the septal areas can be completely lacking to give a characteristic group of lesions which orherwise have normal segmental connections. It is this group of lesions, previously known as "endocardial cushion defects" or "atrioventricular canal malformations", which are best analyzed in terms of deficient atrioventricular septation.

Because there are no atrioventricular septal structures, the abnormal hearts show several characteristic features. Almost always a hole is present at the anticipated site of the septal structures. The hearts are recognizable, however, even when the septum is closed. This is because there is a common atrioventricular junction guarded by a basically common valve. The valve has two leaflets, which bridge the ventricular septum, and a left component with three leaflets. The subaortic outflow tract sits anterosuperior relative to the common atrioventricular junction and there is an abnormal arrangement of the tension apparatus supporting the left atrioventricular valve. There is also gross disproportion between the inlet and outlet dimensions of the septal structure of the left ventricle.

Although all atrioventricular septal defects show these characteristic features, they also show important anatomic differences. These relate, first, to the arrangement of the bridging leaflets themselves. These can either be separate structures, leaving a common atrioventricular orifice, or can be joined by a tongue of tissue dividing the common orifice into separate right and left valvar components. The second feature is the relation of the bridging leaflets to the septal structures. If the bridging leaflets, and connecting tongue if present, are firmly attached to the crest of the ventricular septum, then shunting through the septal defect can only be at atrial level. If the leaflets float, then shunting can occur at both atrial and ventricular levels. If attached to the underside of the atrial septum, shunting can occur only at ventricular level. Further variation is then found in the way the atrioventricular junction is shared between atrial and ventricular masses, giving the arrangements described in terms of ventricular dominance or double outlet atrium. Note must also be taken of important associated malformations, particularly those affecting the left atrioventricular valve, and the abnormal arrangement of the atrioventricular conduction tissues.

\section{Transatrial repair of tetralogy of Fallot in infants}

R. Lamb, J. Gnanapragasam, S. Webber, B. Keeton, A. Salmon and J. Monro

Southampton General Hospital, Southampton

In Southampton the policy of management of children with tetralogy of Fallot has been to perform surgical correction during infancy. Between January 1989 and June 1992 we adopted the transatrial approach and have retrospectively reviewed the results. Nineteen infants, mean age 8.25 months (range 5.25 to 11.25 months), mean weight $6.87 \mathrm{~kg}(4.4-8.9 \mathrm{~kg}$ ), underwent correction for cyanosis (17) and hypercyanotic spells (2). Two had previously undergone modified Blalock-Taussig shunts. 12 had bicuspid pulmonary valves and 2 had an anomalous left anterior descending coronary artery crossing the infundibulum. In all cases both the right ventricular outflow tract obstruction and the ventricular septal defect were dealt with transatrially. The pulmonary valve "annulus" was crossed with a limited incision $(<10 \mathrm{~mm})$ in six, but left intact in 13. Either one or both branch pulmonary arteries were patched in three. All patients are alive and asymptomatic on no medication during a follow-up period of two months to 3.1 years (mean 1.04 years). All are in sinus rhythm, two have hemodynamically insignificant ventricular septal defects detectable only by color Doppler. Residual right ventricular outflow tract gradient $<20 \mathrm{~mm} \mathrm{Hg}$ in 13,20-30 $\mathrm{mm} \mathrm{Hg}$ in four, 60 $\mathrm{mm} \mathrm{Hg}$ in two. In both the later cases the pulmonary valve annulus was not patched and the gradient appears to be at this level. Both await investigation by cardiac catheterization. We conclude that transatrial repair is an acceptable technique for the management of tetralogy of Fallot in infancy.

Repair of tetralogy of Fallot with pulmonary atresia in early infancy H. Uernura, T. Yagihara, F. Yamamoto, K. Nishrguki, O. Matsuki and $Y$. Kawashima

National Cardiovascular Center, Suita

The deleterious effect of prolonged cyanosis on the systemic organs in tetralogy of Fallot with pulmonary atresia may be minimized by definitive repair in early infancy. Transannular patching, instead of using an external conduit, can be usefully applied and would probably avoid future reconstruction of the right ventricular outflow tract. Five patients without systemic-to-pulmonary collateral arteries underwent repair at the age of one to six [mean 3] months old, using transannular patching with a monocusp. Continuity between the right ventricle and pulmonary trunk was achieved by direct anastomosis (in two patients) or interposition of the left atrial appendage (in one), when the atresia was not membranous. All patients were repaired by right ventriculotomy ranging $24-30 \%$ of right ventricular length. All are alive and well. Postoperative pressure studies in the operatung room showed: systolic right ventricle-left ventricle pressure ratio was $0.50-0.63$ [mean 0.54 ], systolic pressure gradient between right ventricle and pulmonary artery $4-25[12] \mathrm{mm}$ $\mathrm{Hg}$, and mean pulmonary arterial pressure $12-18$ [15] $\mathrm{mm} \mathrm{Hg}$. Postoperative (3-15 months) echocardiography showed the velocity in the reconstructed right ventricular outflow tract to be 0.8-1.7 [1.4] $\mathrm{m} / \mathrm{s}$, and pulmonary regurgitation was slight or moderate. The measured values were not inferior to those of patients after repair of tetralogy of Fallot without right ventriculotomy at a comparable age. Tetralogy of Fallot with pulmonary atresia can be repaired during 
early infancy using a transannular patch. Early results are satisfactory.

Prospective study on the indication for balloon dilatation in coarctation of the aorta

T. Ino, S. Shimazaki, K. Akimoto and K. Yabuta

Department of Paediatrics, Juntendo University School of

Medicine, Tokyo

To determine the indication for balloon angioplasty for coarctation of the aorta, the outcome of patients prospectively treated with balloon angioplasty, surgery and drug intervention according to our therapeutic protocol was evaluated. The protocol included postoperative recoarctation and isolated native coarctation of the aorta with operative risks as an absolute indication of balloon angioplasty, and native coarctation without a significant duct as a relative indication. The ductal-dependent coarctation of the aorta complex and diffuse hypoplasia of the aortic arch were contraindications for balloon angioplasty and were treated with surgery. Forty-one patients were entered into this protocol between January 1988 and August 1992. In all, 17 of the 41 patients underwent balloon dilatation angioplasty with a significant pressure gradient relief (from $39 \pm 17$ to $15 \pm 7 \mathrm{~mm} \mathrm{Hg}$ ). Two with native coarctation had restenosis during the period of follow-up and were successfully treated with repeat balloon angioplasty. Out of 14 patients with surgical coarctectomy, 5 died at coarctectomy and 3 died at second corrective surgery. Six of the 10 patients undergoing drug treatment alone died of congestive heart failure. All had chromosomal abnormalities with severe assoctated extracardiac anomalies. The therapeutic strategy for coarctation of the aorta might be changed by use of balloon angioplasty. Our protocol seems to be reasonable, but further evaluation is needed.

Pathological basis of His-bundle tachycardia in four cases Siew Yen Ho and Janice Till

National Heart and Lung Institute, London

His-bundle tachycardia occurring subsequent to surgical repair of congental heart malformations is a life-threatening complication but little is known about its pathological substrate. We have examined the hearts from four patients who developed His-bundle tachycardia following repair of their heart malformations and died within 55 hours of their surgical procedures. The main cardiac lesions were: tetralogy of Fallot (Cases 1 and 2), dysplasia of the atroventricular valves with severe mitral incompetence (Case 3 ) and atrioventricular septal defect (Case 4). Histological studies of the conduction system showed haemorrhage in the environs of the atrioventricular node and penetrating bundle in Cases 1-3 with tracks extending to the proximal branching bundle in Case 2. The haemorrhage was related to sutures placed close to the conduction tissues. In Case 4, haemorrhage affected only the bundle branches but there was direct trauma to the penetrating and common portions of the conduction axis caused by two sutures. The sutures skimmed through the upper part of the bundle. The sinus node was normal in all cases. The pathologic basis of His-bundle tachycardia appeared to be haemorrhagic invasion of the proximal bundle axis in three cases and direct trauma in one case. In all cases, the trauma probably created an irritable focus rather than interrupted the conducrion axis complerely.

Permanent cardiac pacing in paediatric age and in young adults after cardiac surgery for congenital heart disease Roberto Renzi, Maria Grazıa Bottuzzi, Umberto Berrettini, Luigi Ferrini and Angelo Soro

Ospedale G.M. Lancist, Ancona

The intracardiac repair of congenital heart diseases in the paediatric age group implies the risk of alterations in the atrioventricular conduction and in the sinus atrial function which may cause atrioventricular block and sick sinus syndrome. These anomalies, which may have an early or late onset, require non-invasive and/or invasive methods of study in order to be correctly evaluated, to have the best appropriate pacing mode and to avoid complications, more frequent in this range of age. In our experience from 1970 to 1992, 858 parients were operated for congenital heart diseases in G.M. Lancisi Hospital. Fourteen of them (1.7\%) received a permanent pacemaker after the correction because of a high degree atrioventricular block. No patients developed sick sinus syndrome in accord to the common criteria, based on Holter and clinical data, but two patients died suddenly, seven and 12 months, respectively, after a Mustard operation. Age at pacemaker implantation ranged from 43 days to 25 years (mean age 8.9 years). Cardiac defects in the 14 patients were: ventricular septal defect (3 patients), subaortic stenosis (3), atrioventricular septal defect (3), tetralogy of Fallot (3) double outlet right ventricle (1), transposition of the great arteries with ventricular septal defect (1).

The highest frequency of postoperative block was detected after the correction of subaortic stenosis. The pacemaker was implanted in a period ranging from 0 to 36 days after open heart surgery in patients with early onset atrioventricular block and after a mean time of five years in three patients with late onset atrioventricular block. Clinical indications for permanent cardiac pacing were: 1) elective, 2) syncope, 3) congestive heart failure. The site of atrioventricular block was infrahisian in four patients and suprahisian in four among the eight patients studied. The modality of pacing was epicardial VVI rate in 13 patients and endocardial DDD in one patient. The mean follow-up of the 14 patients paced was 12 years. Complications were observed in five cases (37\%). One patient died because of congestive heart failure (DDD); the change in pacing mode was performed in three patients. Epicardial system was changed with endocardial system in two patients. The pacemaker was removed from three patients as they reverted back to stable sinus rhythm with normal atrioventricular conduction. In our experience, two-chambered pacing was the best solution in children operated for congenital heart disease, and the endocardial pacing has to be achieved as soon as possible in every patient.

\section{Interrupted aortic arch-four years' experience in the Singapore General Hospital Wong Keng Yean, Ng Moi Pen and Ong Kim Keat} Cardiology Department, Singapore General Hospital, Singapore Interrupted aortic arch occurs in $<1 \%$ of patients with congenital heart disease. We discuss the clinical presentation, diagnosis, management and outcome of this condition from April 1988 to March 1992 in 10 consecutive patients admitted to the Singapore General Hospital. The age at presentation ranged from 1-14 days (mean-4 days). The weight range was from 2.5 to $4.1 \mathrm{~kg}$ (mean-3 $\mathrm{kg}$ ). There were four males and six females. Clinical presentation included tachypnea, cyanosis, weak pulses, cardiac murmur, and acidosis. Electrocardiogram was non-specific. Chest $\mathrm{x}$-ray showed cardiomegaly and plethora. Diagnosis was made with cross-sectional echocardiography. Associated lesions included patent arterial duct, ventricular septal defect, atrioventricular septal defect, aortopulmonary window and total anomalous pulmonary venous drainage. Four patients had cardiac catheterization which showed Type B interruption. Survival without surgery ranged from nine to 54 days (mean-21). Four patients underwent complete surgical repair with one operative death. Interrupted aortic arch is a fatal condition where early diagnosis and treatment may lead to a favorable outcome. The diagnostic techniques and pitfalls are discussed. 
Common arterial trunk-repair of six cases without using a prosthetic conduit

Taejin Yun, Hee Jong Baek, Jeong Ryul Lee, Yong Jin Kim, Joon Ryang Rho and Kyung Phill Suh

Department of Thoracic \& Cardiovascular Surgery, College of Medicine, Seoul National University, Seoul

Between mid-1990 and July 1992, six patients underwent total surgical correction of common arterial trunk without using a prosthetic conduit. They were five males and one female and their age ranged from two months to 11 months. Anatomic types were type I in four patients, type II in one patient and type III in one patient as determined by the Collett-Edwards classification. Surgical techniques were similar to the first description of Barbero-Marcial, apart for the fact that the distal pulmonary arterial stumps were approximated to the ventriculotomy without need to use the Lecompte manoeuvre in all cases. Monocusps were made using glutaraldehyde fixed autologous pericardium and were placed in the right ventricular outflow tract. Three patients died postoperatively. The causes of death were acute myocardial failure in one patient, low cardiac output in another patient and lung complication due to pulmonary vascular disease in the third patient. The three survivors have been followed for four to seven months without significant complications.

Pulmonary atresia with intact ventricular septum

Walid Dihmis, Rob Parry, Peter Wilde, Janardan Dhasmana, Steve Jordan and James Wisheart

Bristol Royal Infirmary, Bristol

Between 1975 and 1981,15 patients presented with pulmonary atresia and intact ventricular septum of whom 11 died (73\%). A review of this condition emphasized variations in the morphology of the right ventricle and the size of the tricuspid valve which led to a programme of staged surgical correction:

STAGE I: Neonatal closed palliation, usually Blalock-Taussig shunt. STAGE II: Open palliation by right ventricular outflow tract reconstruction.

STAGE III: Definitive open surgery by either correction or Fontan procedure.

The results of this policy in babies presenting berween 1982 and 1990 have been studied by a retrospective review. Operative mortality is death within 30 days.

Twenty-two babies presented with pulmonary atresia with intact ventricular septum. The parents of two declined trearment, leaving 20 available for study.

STAGE I: Neonatal closed palliation in 20 patients; 17 survivors, of whom 4 required a second palliative operation with 1 death, 12 proceeded to Stage II, 2 directly to Stage III and 2 await further treatment.

STAGE II: Open palliation - 12 cases; 10 survivors of whom 8 proceeded to Stage III. 1 late death

STAGE III: Definitive surgery - 10 cases. Correction in 8 with no deaths; Fontan operation in 2 with 1 death.

Of 20 patients with pulmonary atresia with intact ventricular septum, 13 are still alive, 9 having completed the programme successfully, while 2 await Stage III. For the whole group the mortality for pulmonary atresia with intact ventricular septum has fallen from $73 \%$ prior to 1982 to $35 \%$. We continue to advocate a policy of staged repair for pulmonary atresia with intact ventricular septum.

Pulmonary artery sling-tracheal resection and repositioning without vascular anastomosis F. Splittgerber,' R. Liersch, ${ }^{2} K$. Winter, ${ }^{2}$ B. Falk and $^{\prime}$. Minale' From the Department of Cardiovascular and Thoracic Surgery' and Paediatric Cardiology, ${ }^{2}$ Wuppertal City Hospital, Wuppertal

The standard operative procedure for left pulmonary artery (PA) sling consists of left PA resection and reimplantation anterior to the trachea, often combined with tracheopexy. This will frequently leave a tracheal stenosis, which may necessitate additional later interventions. An alternate method is tracheal resection at the level of the aberrant pulmonary artery and tracheal reanastomosis behind the PA after removal of the tracheomalacic segment. ${ }^{1,2}$

In 1992 we operated upon a 5 months old girl with left pulmonary artery sling. The child suffered from severe respiratory distress due to tracheomalacia at the level of the aberrant left PA. The operation was performed with cardiopulmonary bypass and an episode of profound hypothermic circulatory arrest. The left PA was dissected free; $0.5 \mathrm{~cm}$ of malacic trachea were resected and primary tracheal reanastomosis performed behind the PA using running 5-0 PDS suture. The child was extubated on the first postoperative day. The hospital course was uncomplicated. Three months postoperatively the child was completely asymptomatic.

In comparison to pulmonary arterial resection, this method of tracheal resection is an advantageous alternate method for the treatment of pulmonary artery sling. Since the tracheomalacia is the main culprit, it appears best to correct this condition directly. A normal anatomic relationship between left PA and trachea can be established without need for any vascular anastomosis.

1. Jonas RA; Spevak PJ, McGill T, Castañeda AR. Pulmonary artery sling: primary repair by tracheal resection in infancy. J Thorac Cardiovasc Surg 1989; 97: 548-50.

2. Wu MH, Lai WW. Sleeve resection of congenital tracheal stenosis associated with pulmonary artery sling: report of a case. Taiwan I Haueh Hui Tsa Chih 1990; 89: 724-726.

Pacemaker management in congenital complete heart block in infancy

Wong Keng Yean, Teo Wee Siong and Ong Kim Kiat

From the Department of Cardiology, Singapore General Hospital, Singapore

Epicardial pacemakers were implanted in 3 infants with congenitally complete heart block over the period November to June 1992 in the Singapore General Hospital. The first patient was 13 months old. Mother has SLE. Poor left ventricular function with moderate mitral regurgitation. Developed left hemiplegia post-implantation. The second parient was 9 days old and weighed $2.6 \mathrm{~kg}$. Diagnosis was made antenatally and there was early cardiac failure. The third patient was 1 day old and weighed $3.6 \mathrm{~kg}$. Morher was anti-Ropositive. Diagnosis was made antenatally and again there was early cardiac failure. At periods of follow-up of 3 years, 10 months and 4 months, respectively, all patients are well. Congenitally complete heart block in infancy may require management by insertion of pacemaker. We discuss our early experience in decision-making, preoperative preparation, post-operative complication, and follow-up.

Surgical treatment of cor triatriatum Young Tae Kim, Jong Meun Hong, Jeong Ryul Lee, Yong Jin Kim, Joon Ryang Rho and Kyung Phill Suh From the Department of Thoracic and Cardiovascular Surgery, College of Medicine, Seoul National University, Seoul From April 1981 to April 1992, 24 patients with cor triatriatum were treated at Seoul National University Hospital. Ages ranged from 1 month to 24 years with mean of 7.4 years.

Twenty patients had interatrial communications through a patent foramen ovale, primum or secundum defect of the atrial septum. Four had no interatrial communications. Fourteen patients had associated anomalies: partial anomalous pulmonary venous connection in 3 , total anomalous pulmonary venous connection in 2 , persistent left superior caval vein in 3 , and other anomalies in 6 patients. Surgical corrections were performed through right atriotomy 
in 18 , left atriotomy in 4 , and both atriotomy in 2 patients. Three patients (12.5\%) died early after operation: two of them were associated with single ventricle. Six out of 21 survivors (28.6\%) experienced complications: recurrent pneumonı, pulmonary embolism, ischemic encephalopathy, diaphragmatic palsy and tachyarrhythmias. At the time of follow-up all survivors, except one, were in functional class 1 .

Surgical correction of cor triatriatum restored normal hemodynamic status with relatively low operative mortality, especially in patients not complicated with severe anomalies.

\section{Effect of diltiazem on graft vascular disease in pediatric heart transplants \\ Hersch Rosenberg, Alan Menkis, Richard Novick and Neil \\ McKenzie \\ Children's Hospital of Western Ontario, University of Western \\ Ontario, London, Canada}

Currently, one of the major limitations to heart transplantation has been the early development of graft vascular disease. Pediatric recipients may be particularly predisposed to this complication. To assess the impact of diltiazem therapy on the development of graft vascular disease, we retrospectively analyzed a series of 10 patients undergoing cardiac transplantation between 1988 and early 1992. All patients were followed for a minimum of 6 months. Five children received diltiazem (Group 1). Five children not receiving diltiazem served as controls (Group 2). Age at transplant (Group 1-9.6 years \pm 6.2 versus Group $2-5.1$ years \pm 3.5 ) and duration of follow-up (Group 1-11.4 months \pm 8.4 versus Group 2-13.0 months \pm 6.2 ) were comparable in both groups. No child receiving diltiazem died of vascular disease versus 3 of 5 children not receiving the drug $(\mathrm{p}<.05)$. These results suggest that diltiazem may have an ameliorating effect on graft vascular disease following cardiac transplantation and merits further study.

\section{Congenital coronary artery fistulas}

Jong Ho Park, Jeong Ryul Lee, Yong Jin Kim, Joon Ryang Rho, and Kyung Phill Suh

From the Department of Thoracic and Cardiovascular Surgery, Seoul National University Hospital, Seoul

From 1981 to 1991 , we experienced 25 patients with congenital coronary arteriovenous fistulas at our institution. A retrospective review was made to delineate the course and the management of coronary arteriovenous fistulas and to clarify the role of surgical treatment. Fifteen patients were male and 10 were female with a mean age of 17.4 years (from 3 months to 58 years). The most frequent symptom was dyspnea on exertion $(56 \%)$. Other symptoms were congestive heart failure, angina and palpitation; $64 \%$ of the patients were sympromatic, $47 \%$ of patients less than 20 years old were symptomatic and $100 \%$ of patients over 20 years old were symptomatic. Three patients had multuple coronary arteriovenous fistulas. The fistula drained into the right ventricle in 13, pulmonary trunk in 1, left ventricle in 4, right atrium in 2 , and left atrium in 1. 13 patients had other associated cardiac lesions. Mean pulmonaryto-systemic flow $\left(Q_{/} / Q\right)$ in the isolate coronary arteriovenous fistula group was 2.19 . All patients were operated on to correct the fistulas and other associated cardiac lesions. Every patient had follow-up from 1 month to 11 years without late death. Postoperative complication rate was $24 \%$ - significant arrhythmia (3), recurred coronary arteriovenous fistulas (1), psychosis (1), pneumonia (1). Symptom improvement was evident postoperatively. Below 20 years old, $94 \%$ of patients were asymptomatic, but above 20 years old, symptoms persisted in $25 \%$. In summary, early elective ligation of coronary arteriovenous fistulas was indicated in all patients because of the high incidence of late symptoms and complications and the increased morbidity associated with ligation in older patients.

A method to determine optimal anastomotic geometries in right heart bypass surgery in an animal model

Sheri L. Carroll,' Giancarlo Crupi, ${ }^{2}$ Robert Kreski, ${ }^{1}$ James A. Thompson, Jose I. Ferreiro,' Carol L. Lucas' and G. William Henry'

From The University of North Carolina, ${ }^{\prime}$ Chapel Hill, and The Ospedalt Riuntti, ${ }^{2}$ Bergamo

Right heart bypass techniques are used to restore systemic venous return to the pulmonary circulation in multiple congenital heart defects (e.g., absent right atrioventricular connection) as a primary repair or following palliative procedures (e.g., cavopulmonary connections). Different anastomotic connections (e.g., Fontan procedure) result in varied geometries. The optimal connection would promote the most efficien $\mathrm{c}$ flow from the venous return to the pulmonary circulation. This study presents a method to describe patterns of blood flow associated with right heart bypass procedures to determine the optimal anastomotic geometries by using an in vitro-derived accurate anatomic model. An anatomically accurate, acrylic flow-through model can be constructed from a flexible silicone cast obtained in situ following different right heart bypass procedures. The model can then be used to visualize flow patterns to determine the effect of geometry on such patterns. This method is demonstrated in the study of an anastomosis between the pulmonary trunk and right atrial appendage combined with an end-to-side cavopulmonary anastomosis in an ovine model.

\section{Flow visualization in an in vitro model of a Blalock-Taussig} anastomosis

James A. Thompson, Sher L. Carroll, Carol L. Lucas, Jose Ferreiro, G. William Henry

From the University of North Carolina, Chapel Hill

The Blalock-Taussig anastomosis and its modified variants are palliative procedures used frequently in cyanotic congenital heart disease. This study proposes a method to observe the flow patterns created by surgical modification of the pulmonary circulation. An $t n$ vitro, clear acrylic flow-through model simulating the anatomy of a modified Blalock-Taussig shunt was constructed. This model was then used to visualize the patterns created by pulsatile.flow at various physiologic rates. Vortices and areas of flow separation were observed at the entrance to the shunt, with in the shunt, and at the anastomosis to the pulmonary arteries. The model used for this study was then modified to increase variables, for example, by incorporating competing flow from the pulmonary trunk, changing resistances, and altering geometries of shunt anastomoses, etc. The optimal surgical connection would result in the most efficient flow of blood from the systemic to the pulmonary circulation; experiments are currently being done to evaluate the loss of flow energy across surgical shunts. The knowledge gained from this type of experimentation was further advanced by constructing anatomically accurate flow-through models of surgically altered pulmonary circulations, which more closely approximate an in vivo system.

Blood flow velocity profiles in pulmonary branch arteries in lambs Katayama H, Ferreiro JI, Lucas CL, Ha B, Frantz EG and Henry GW

\section{The University of North Carolina, Chapel Hill}

We studied the detailed profiles of blood flow in the right and left pulmonary arteries using $20 \mathrm{MHz}$ pulsed Doppler ultrasound equipment in a lamb model. Fourteen lambs aged four to six weeks were selected. In six lambs, monocrotaline pyrrole was injected parentally to create pulmonary hypertension ( $\mathrm{PH}$ group). Eight other lambs served as unaltered controls (control group). The blood flow velocities were sampled in $1 \mathrm{~mm}$ increments along the anterior- 
posterior axis of the branch arteries. The maximum velocity of the forward flow in the left pulmonary artery was higher than that in the right pulmonary artery in the control group $(71.7 \pm 15.9 \mathrm{~cm} / \mathrm{s}$ vs $60.2 \pm 13.5 ; p<0.05)$. The fastest backward flow was located at the posterior position of the vessel in the right pulmonary artery in the control group. No significant bias in location was shown in the left pulmona ry artery. Using indices of $\mathrm{P90}$, acceleration time (AcT), and $\mathrm{P} 90 \mathrm{AcT}$, the velocity waveforms in the $\mathrm{PH}$ group were compared with those in the control group. In the left pulmonary artery, every index in the control group showed a significantly greater value than that in the $\mathrm{PH}$ group. On the other hand, no significant differences were found between either group in the right pulmonary artery.

\section{Echo' '93-Pediatric Echocardiography Congress \\ April 22-24 \\ Naples, Italy}

\begin{tabular}{|c|c|c|c|}
\hline \multirow[t]{2}{*}{ TOPICS } & \multicolumn{3}{|c|}{$\begin{array}{l}\text { Preoperative assessment, perioperative assessment, long-term } \\
\text { follow-up of: }\end{array}$} \\
\hline & \multicolumn{3}{|c|}{$\begin{array}{l}\text { Fontan and Glenn operations } \\
\text { Valvar and subvalvar aortic stenosis } \\
\text { Pulmonary atresia with intact ventricular septum } \\
\text { Complex transposition of the great arteries }\end{array}$} \\
\hline \multirow[t]{3}{*}{ FACULTY } & $\begin{array}{l}\text { P. Arciprete } \\
\text { L. Ballerini } \\
\text { M. Carminati } \\
\text { M. Chiariello } \\
\text { M. Cotrufo } \\
\text { G. Crupi } \\
\text { A. Dagianti } \\
\text { L. Daliento, }\end{array}$ & $\begin{array}{l}\text { F. de Leva } \\
\text { G. di benedetto } \\
\text { A. lacono } \\
\text { A. Ludomirsky } \\
\text { C. Marcelletti } \\
\text { B. Marino } \\
\text { G. Pacileo } \\
\text { L. Parenzan } \\
\text { L. Pasquini }\end{array}$ & $\begin{array}{l}\text { F. Picchio } \\
\text { S.B. Ritter } \\
\text { P. Rizzon } \\
\text { M.G. Rusco } \\
\text { R. A. Snider } \\
\text { V. Sperandeo } \\
\text { G. Thiene } \\
\text { C. Vosa }\end{array}$ \\
\hline & \multicolumn{3}{|c|}{$\begin{array}{r}\text { Poster abstract forms can be sent to the Organizing Secretariat until } \\
\text { 30 March } 1993\end{array}$} \\
\hline & & & $\begin{array}{r}\text { or general information call } \\
\text { Raffaele Calabrò, M.D. } \\
\text { Pediatric Cardiology } \\
\text { Ospedale Monald } \\
\text { Via L. Bianch } \\
80131 \text { Napoli, Italy } \\
\text { Tel. 39-81-706-2330 } \\
\text { Fax. 39-81-706-2355 }\end{array}$ \\
\hline
\end{tabular}

